IFT-P.072/2001

KCL-TH-01-49

hep-th/0112160

October 22, 2018

\title{
Ten-Dimensional Supergravity Constraints from the Pure Spinor Formalism for the Superstring
}

\author{
Nathan Berkovits \\ Instituto de Física Teórica, Universidade Estadual Paulista \\ São Paulo, Brasil \\ and \\ Paul Howe \\ Department of Mathematics \\ King's College, London
}

\begin{abstract}
It has recently been shown that the ten-dimensional superstring can be quantized using the BRST operator $Q=\oint \lambda^{\alpha} d_{\alpha}$ where $\lambda^{\alpha}$ is a pure spinor satisfying $\lambda \gamma^{m} \lambda=0$ and $d_{\alpha}$ is the fermionic supersymmetric derivative. In this paper, the pure spinor version of superstring theory is formulated in a curved supergravity background and it is shown that nilpotency and holomorphicity of the pure spinor BRST operator imply the on-shell superspace constraints of the supergravity background. This is shown to lowest order in $\alpha^{\prime}$ for the heterotic and Type II superstrings, thus providing a compact pure spinor version of the ten-dimensional superspace constraints for $N=1$, Type IIA and Type IIB supergravities. Since quantization is straightforward using the pure spinor version of the superstring, it is expected that these methods can also be used to compute higher-order $\alpha^{\prime}$ corrections to the ten-dimensional superspace constraints.
\end{abstract}




\section{Introduction}

For many purposes, superstring theory is most conveniently expressed as an effective field theory of its massless modes consisting of supergravity theory together with corrections arising order by order in $\alpha^{\prime}$. In principle these higher order corrections can be obtained by computing scattering amplitudes or by demanding consistency of the superstring sigma model in a curved background. However, neither of these procedures is easy to carry out in superstring theory in a way in which spacetime supersymmetry is guaranteed. In the Ramond-Neveu-Schwarz (RNS) formalism, it is difficult to introduce fermionic or Ramond-Ramond background fields, while the Green-Schwarz (GS) formalism ensures spacetime supersymmetry but is difficult to quantise. Although the hybrid formalism for the superstring can be used to compute $\alpha^{\prime}$ corrections in a manner which manifestly preserves $\mathrm{D}=2[1], \mathrm{D}=4[2]$, or $\mathrm{D}=6[3]$ super-Poincaré covariance, one needs a $\mathrm{D}=10$ covariant formalism if one wants to describe the superstring in arbitrary supergravity backgrounds.

In this situation, one might try to study the constraints that ten-dimensional supersymmetry imposes on higher-order contributions to the effective action. One difficulty here is that, with the exception of the supergravity sector of the heterotic string, it is not known how to construct any superspace actions due to the absence of any known sets of auxiliary fields. Even in the heterotic case, the auxiliary fields are rather complicated [4] and it is not clear how to construct higher order actions which correspond to superstring corrections, although the $R^{4}$ invariant was discussed from this point of view in [5]. It seems that additional input apart from supersymmetry is required. On the other hand, it has been possible to obtain information about some particular terms, for example in the work of $[6,7,8,9,10,11,12]$. Other approaches to the problem have involved supersymmetrisation of bosonic sigma model terms [13] for the heterotic string [14, 15], and studying corrections to heterotic superspace constraints directly [16, 17], which has at least been successful in incorporating anomaly terms. This work has been reviewed in [18] where string results were used to partially construct $R^{4}$ corrections in M-theory. Other recent approaches to supersymmetrizing the $R^{4}$ term in M-theory are described in [19] and [20].

The fact that one is forced to look at the equations of motion rather than Lagrangians suggests that a way forward might be to understand the geometry behind these equations. Many years ago, Witten showed how the $\mathrm{N}=1 \mathrm{D}=10$ superspace Yang-Mills equations can be understood in terms of integrability along light-like lines and how this is related to $\kappa$-symmetry of the superparticle action [21,22]. This sort of analysis was subsequently carried out for the heterotic [23] and IIB strings [24], and reinterpreted in terms of light-like integrability in loop superspace in $[25,26]$, at least for the heterotic case.

In some related work, one of the present authors showed that light-like integrability could be replaced by integrability along pure spinor lines, and that this can also be employed in eleven dimensions in the context of the supermembrane [27]. A virtue of this approach is that it is simpler than light-like integrability, but, at the time, it wasn't entirely clear how it was related to particle or string actions. More recently, the other author has shown that ten-dimensional superparticles and superstrings can be effectively quantised using pure spinor 
variables $[28,29]$. These pure spinor variables can be interpreted as bosonic ghosts for a fermionic symmetry, although it is not currently fully understood how this can be implemented in a worldsheet reparameterization invariant fashion. Nevertheless, the final gauge-fixed action does have manifest spacetime supersymmetry and correctly fixes the central charge to be zero. Unlike the GS formalism, however, the pure spinor formalism has the tremendous advantage that it can be quantised straightforwardly since the action is free in a flat background.

In this pure spinor formalism [29], the left-moving BRST operator for the heterotic superstring is

$$
Q=\oint \lambda^{\alpha} d_{\alpha}
$$

where $\lambda^{\alpha}$ is a bosonic pure spinor variable satisfying ${ }^{1}$

$$
\lambda^{\alpha} \gamma_{\alpha \beta}^{m} \lambda^{\beta}=0
$$

for $m=0$ to 9 , and $d_{\alpha}$ is the worldsheet variable corresponding to the $\mathrm{N}=1 \mathrm{D}=10$ spacetime supersymmetric derivative. In a flat background, $\lambda^{\alpha}$ and $d_{\alpha}$ are holomorphic and $d_{\alpha}$ satisfies the OPE $d_{\alpha}(y) d_{\beta}(z) \rightarrow-i \alpha^{\prime}(y-z)^{-1} \gamma_{\alpha \beta}^{m} \Pi_{m}$ where $\Pi_{m}=\partial x_{m}+\frac{i}{2} \theta \gamma_{m} \partial \theta$ is the supersymmetric momentum. So $\lambda \gamma^{m} \lambda=0$ implies that $Q$ is nilpotent. A natural conjecture is that in a curved supergravity/super-Yang-Mills background for the heterotic superstring, nilpotence and holomorphicity of $\lambda^{\alpha} d_{\alpha}$ implies the superspace equations of motion for the background superfields.

Similarly, in the pure spinor formalism for the Type II superstring, the left and right-moving BRST operators are ${ }^{2}$

$$
Q=\oint \lambda^{\alpha} d_{\alpha}, \quad \bar{Q}=\oint \hat{\lambda}^{\hat{\alpha}} \hat{d}_{\hat{\alpha}}
$$

where $\lambda^{\alpha}$ and $\hat{\lambda}^{\hat{\alpha}}$ are independent pure spinor variables satisfying

$$
\lambda^{\alpha} \gamma_{\alpha \beta}^{m} \lambda^{\beta}=0, \quad \hat{\lambda}^{\hat{\alpha}} \gamma_{\hat{\alpha} \hat{\beta}^{m}}^{m} \hat{\lambda}^{\hat{\beta}}=0,
$$

for $m=0$ to 9 , and $d_{\alpha}$ and $\hat{d}_{\hat{\alpha}}$ are worldsheet variables corresponding to the $\mathrm{N}=2 \mathrm{D}=10$ spacetime supersymmetric derivatives. In a flat background, $\lambda^{\alpha} d_{\alpha}$ is holomorphic and nilpotent whereas $\hat{\lambda}^{\hat{\alpha}} \hat{d}_{\hat{\alpha}}$ is antiholomorphic and nilpotent. So it is natural to conjecture that in a curved $\mathrm{N}=2 \mathrm{D}=10$ supergravity background for the Type II superstring, the superspace equations of motion for the background are implied by the condition that these properties of $\lambda^{\alpha} d_{\alpha}$ and $\hat{\lambda}^{\hat{\alpha}} \hat{d}_{\hat{\alpha}}$ are preserved.

In this paper, we shall verify the above conjectures to lowest order in $\alpha^{\prime}$ for the heterotic and Type II superstrings in $N=1$ and $N=2$ supergravity backgrounds. This verification will lead to new pure spinor versions of the superspace constraints for ten-dimensional $N=1$, Type IIA and Type IIB supergravity. These have the property that they are remarkably compact and may be useful for studying other aspects of ten-dimensional supersymmetric theories such as

\footnotetext{
${ }^{1}$ We will use the notation where $\gamma_{\alpha \beta}^{m}$ and $\gamma^{m \alpha \beta}$ are $16 \times 16$ symmetric matrices which form the off-diagonal blocks of the $32 \times 32$ ten-dimensional $\Gamma$-matrices in the Weyl representation.

${ }^{2}$ Throughout this paper, we will use spinor notation simultaneously for the Type IIA and Type IIB superstring by imposing that $\alpha$ and $\hat{\alpha}$ denote $\mathrm{D}=10$ spinors of opposite chirality for the IIA superstring and denote spinors of the same chirality for the IIB superstring.
} 
harmonic superspace. Furthermore, since the superstring action is quantizable, this conjecture can be used in principle to compute the superspace equations of motion to arbitrary order in $\alpha^{\prime}$.

For the $\mathrm{N}=1 \mathrm{D}=10$ supergravity/super-Yang-Mills background of the heterotic superstring, nilpotence of $\lambda^{\alpha} d_{\alpha}$ will imply

$$
\lambda^{\alpha} \lambda^{\beta} T_{\alpha \beta}{ }^{C}=\lambda^{\alpha} \lambda^{\beta} H_{\alpha \beta C}=\lambda^{\alpha} \lambda^{\beta} F_{\alpha \beta}^{I}=0
$$

where $T_{A B}{ }^{C}, H_{A B C}$ and $F_{A B}^{I}$ are the superspace torsion, three-form field strength and superYang-Mills field strength. These equations are identical to those derived from pure spinor integrability in [27]. Since (5) must be satisfied for an arbitrary pure spinor $\lambda^{\alpha}$ satisfying (2), (5) implies that

$$
\left(\gamma_{m n p q r}\right)^{\alpha \beta} T_{\alpha \beta}{ }^{C}=\left(\gamma_{m n p q r}\right)^{\alpha \beta} H_{\alpha \beta C}=\left(\gamma_{m n p q r}\right)^{\alpha \beta} F_{\alpha \beta}^{I}=0
$$

for any self-dual five-form direction mnpqr. Up to conventional constraints (which will be implied by holomorphicity of $\lambda^{\alpha} d_{\alpha}$ ), the constraints of (6) will be shown to imply the standard $\mathrm{N}=1$ supergravity/super-Yang-Mills equations of motion.

For the $\mathrm{N}=2 \mathrm{D}=10$ supergravity background of the Type II superstring, nilpotence of $\lambda^{\alpha} d_{\alpha}$ and $\hat{\lambda}^{\hat{\alpha}} \hat{d}_{\hat{\alpha}}$ will imply

$$
\begin{aligned}
& \lambda^{\alpha} \lambda^{\beta} T_{\alpha \beta}{ }^{C}=\hat{\lambda}^{\hat{\alpha}} \hat{\lambda}^{\hat{\beta}} T_{\hat{\alpha} \hat{\beta}}{ }^{C}=\lambda^{\alpha} \hat{\lambda}^{\hat{\beta}} T_{\alpha \hat{\beta}}{ }^{C}=0, \\
& \lambda^{\alpha} \lambda^{\beta} H_{\alpha \beta C}=\hat{\lambda}^{\hat{\alpha}} \hat{\lambda}^{\hat{\beta}} H_{\hat{\alpha} \hat{\beta} C}=\lambda^{\alpha} \hat{\lambda}^{\hat{\beta}} H_{\alpha \hat{\beta} C}=0 .
\end{aligned}
$$

Since $\lambda^{\alpha}$ and $\hat{\lambda}^{\hat{\alpha}}$ are arbitrary pure spinors satisfying (4), (7) implies that

$$
\begin{aligned}
& \left(\gamma_{m n p q r}\right)^{\alpha \beta} T_{\alpha \beta}{ }^{C}=\left(\gamma_{m n p q r}\right)^{\hat{\alpha} \hat{\beta}} T_{\hat{\alpha} \hat{\beta}}{ }^{C}=T_{\alpha \hat{\beta}}{ }^{C}=0, \\
& \left(\gamma_{m n p q r}\right)^{\alpha \beta} H_{\alpha \beta C}=\left(\gamma_{m n p q r}\right)^{\hat{\alpha} \hat{\beta}} H_{\hat{\alpha} \hat{\beta} C}=H_{\alpha \hat{\beta} C}=0
\end{aligned}
$$

for any self-dual five-form direction mnpqr. Up to conventional constraints (which will be implied by holomorphicity and antiholomorphicity of $\lambda^{\alpha} d_{\alpha}$ and $\hat{\lambda}^{\hat{\alpha}} \hat{d}_{\hat{\alpha}}$ ), the constraints of (8) will be shown to imply the standard Type II supergravity equations of motion. ${ }^{3}$

In section 2 of this paper we shall use the heterotic superstring sigma model to derive a pure spinor version of the $N=1$ supergravity/super-Yang-Mills constraints, and in section 3 we shall use the Type II superstring sigma model to derive a pure spinor version of the Type IIA and Type IIB supergravity constraints. In section 4 the pure spinor description of Type IIB supergravity will be shown to agree with the standard Howe-West (HW) superspace description of [30]. In section 5 we shall briefly discuss the the procedure for extending to higher order in $\alpha^{\prime}$ these computations of the ten-dimensional superspace constraints.

\footnotetext{
${ }^{3}$ As will be explained in section 3 , the superspace torsion $T_{A B}{ }^{C}$ appearing in (7) and (8) is not the usual one since some of its components depend on a "left-moving" spin connection and some of its components depend on a "right-moving" spin connection.
} 


\section{Heterotic Superstring Sigma Model}

In this section, the pure spinor version of the heterotic superstring will be reviewed in flat and curved backgrounds. Nilpotence and holomorphicity of $\lambda^{\alpha} d_{\alpha}$ will then be shown to imply the superspace equations of motion for the supergravity/super-Yang-Mills background.

\subsection{Heterotic superstring in a flat background}

In the pure spinor version of the heterotic superstring, the worldsheet variables consist of the $N=1 D=10$ superspace variables $\left(x^{m}, \theta^{\alpha}, p_{\alpha}\right)$ for $m=0$ to 9 and $\alpha=1$ to 16 where $p_{\alpha}$ is the conjugate momentum to $\theta^{\alpha}$, as well as the left-moving pure spinor ghost variable $\lambda^{\alpha}$ and its conjugate momentum $w_{\alpha}$, the $E_{8} \times E_{8}$ or $S O(32)$ right-moving currents $\bar{J}^{I}$, and $(\bar{b}, \bar{c})$ right-moving Virasoro ghosts. Because $\lambda^{\alpha}$ is defined to satisfy (2), it has only eleven independent degrees of freedom and its conjugate momentum $w_{\alpha}$ is only defined up to the gauge transformation $\delta w_{\alpha}=\Lambda_{m}\left(\gamma^{m} \lambda\right)_{\alpha}$ for any $\Lambda^{m}$. This gauge transformation can be used to eliminate five components of $w_{\alpha}$, so both $\lambda^{\alpha}$ and $w_{\alpha}$ have eleven independent components.

The action and stress-tensor in a flat background is

$$
\begin{gathered}
S=\frac{1}{2 \pi \alpha^{\prime}} \int d^{2} z\left(\frac{1}{2} \partial x^{m} \bar{\partial} x_{m}+p_{\alpha} \bar{\partial} \theta^{\alpha}+\bar{b} \partial \bar{c}\right)+S_{\lambda}+S_{J}, \\
T=\frac{1}{\alpha^{\prime}}\left(-\frac{1}{2} \partial x^{m} \partial x_{m}-p_{\alpha} \partial \theta^{\alpha}\right)+T_{\lambda}, \quad \bar{T}=\frac{1}{\alpha^{\prime}}\left(-\frac{1}{2} \bar{\partial} x^{m} \bar{\partial} x_{m}-\bar{b} \bar{\partial} \bar{c}-\bar{\partial}(\bar{b} \bar{c})\right)+T_{J},
\end{gathered}
$$

where $S_{\lambda}$ and $S_{J}$ are the actions for $\lambda^{\alpha}$ and $J^{I}$, and $T_{\lambda}$ and $T_{J}$ are the $c=22$ and $c=16$ stress tensors for $\lambda^{\alpha}$ and $J^{I}$. As described in [29], one can write explicit expression for $S_{\lambda}$ and $T_{\lambda}$ by solving the constraint of (2) in terms of eleven chiral bosons $\left(\gamma, u_{a b}\right)$ and their conjugate momenta $\left(\beta, v^{a b}\right)$ where $a=1$ to 5 and $u_{a b}=-u_{b a}$. However, these explicit expressions will not be necessary here. We will only need to know that $S_{\lambda}$ is defined such that $\lambda^{\alpha}$ has no singular OPE with itself and Lorentz currents $N^{m n}$ can be constructed out of $\lambda^{\alpha}$ and its conjugate momentum $w_{\alpha}$ as $N^{m n}=\frac{1}{2 \alpha^{\prime}} \lambda \gamma^{m n} w$ which satisfy the OPE's

$$
\begin{gathered}
N^{m n}(y) \lambda^{\alpha}(z) \rightarrow \frac{1}{2}\left(\gamma^{m n}\right)^{\alpha}{ }_{\beta} \frac{\lambda^{\beta}(z)}{y-z} \\
N^{k l}(y) N^{m n}(z) \rightarrow \frac{\eta^{m[l} N^{k] n}(z)-\eta^{n[l} N^{k] m}(z)}{y-z}-3 \frac{\eta^{k n} \eta^{l m}-\eta^{k m} \eta^{l n}}{(y-z)^{2}} .
\end{gathered}
$$

Similarly, the explicit expression for $S_{J}$ will not be necessary and we will only need to know the OPE

$$
J^{I}(y) J^{K}(z) \rightarrow \frac{\delta^{I K}}{(y-z)^{2}}+f_{L}^{I K} \frac{J^{L}(z)}{(y-z)}
$$

where $f_{L}^{I K}$ are the $E_{8} \times E_{8}$ or $S O(32)$ structure constants.

Physical states of the superstring are defined as vertex operators in the cohomology of the left and right-moving BRST operators

$$
Q=\oint \lambda^{\alpha} d_{\alpha}, \quad \bar{Q}=\oint(\bar{c} \bar{T}+\bar{c} \bar{\partial} \bar{c} \bar{b})
$$


where

$$
d_{\alpha}=p_{\alpha}-\frac{i}{2} \gamma_{\alpha \beta}^{m} \theta^{\beta} \partial x_{m}+\frac{1}{8} \gamma_{\alpha \beta}^{m} \gamma_{m} \gamma \delta \theta^{\beta} \theta^{\gamma} \partial \theta^{\delta} \quad \text { and } \quad \Pi^{m}=\partial x^{m}+\frac{i}{2} \theta \gamma^{m} \partial \theta
$$

are spacetime supersymmetric and satisfy the OPE's [32]

$$
d_{\alpha}(y) d_{\beta}(z) \rightarrow-i \alpha^{\prime}(y-z)^{-1} \gamma_{\alpha \beta}^{m} \Pi_{m}(z), \quad d_{\alpha}(y) \Pi^{m}(z) \rightarrow i \alpha^{\prime}(y-z)^{-1} \gamma_{\alpha \beta}^{m} \partial \theta^{\beta}(z) .
$$

To construct the sigma model for the heterotic superstring, it will be useful to know the integrated form of the massless supergravity and super-Yang-Mills vertex operators, $V_{S G}$ and $V_{s Y M}$, which are

$$
\begin{gathered}
V_{S G}=\int d^{2} z\left(\partial \theta^{\alpha} A_{\alpha m}(x, \theta)+\Pi^{n} A_{n m}(x, \theta)+d_{\alpha} E_{m}^{\alpha}(x, \theta)+\frac{1}{2} N_{n p} \Omega_{m}^{n p}(x, \theta)\right) \bar{\partial} x^{m}, \\
V_{s Y M}=\int d^{2} z\left(\partial \theta^{\alpha} A_{\alpha I}(x, \theta)+\Pi^{n} A_{n I}(x, \theta)+d_{\alpha} W_{I}^{\alpha}(x, \theta)+\frac{1}{2} N_{n p} U_{I}^{n p}(x, \theta)\right) \bar{J}^{I},
\end{gathered}
$$

where $N^{n p}$ are the Lorentz currents for the pure spinor. Note that the first two terms in $V_{S G}$ and $V_{S Y M}$ are the same as in the Green-Schwarz heterotic superstring vertex operators, but the third and fourth terms are needed for the vertex operators to be BRST invariant. These two vertex operators can be obtained by taking the "product" of a massless open superstring vertex operator,

$$
V_{\text {open }}=\int d z\left(\partial \theta^{\alpha} A_{\alpha}(x, \theta)+\Pi^{n} A_{n}(x, \theta)+d_{\alpha} W^{\alpha}(x, \theta)+\frac{1}{2} N_{n p} U^{n p}(x, \theta)\right),
$$

with either $\int d \bar{z} \bar{\partial} x^{m}$ or $\int d \bar{z} \bar{J}^{I}$.

Using the fact that $\lambda^{\alpha} \lambda^{\beta}$ is proportional to $\gamma_{m n p q r}^{\alpha \beta}\left(\lambda \gamma^{m n p q r} \lambda\right)$ and the OPE's of (11) and (16), one can check that $Q V_{S G}=\bar{Q} V_{S G}=0$ implies that

$$
\begin{gathered}
\gamma_{n p q r s}^{\alpha \beta} D_{\alpha} A_{\beta m}=0, \quad \partial^{m}\left(\partial_{m} A_{\beta n}-\partial_{n} A_{\beta m}\right)=0, \\
A_{n m}=-\frac{i}{8} D_{\alpha} \gamma_{n}^{\alpha \beta} A_{\beta m}, E_{m}^{\beta}=-\frac{i}{10} \gamma^{n \alpha \beta}\left(D_{\alpha} A_{n m}-\partial_{n} A_{\alpha m}\right), \Omega_{m}^{n p}=\frac{1}{8} D_{\alpha}\left(\gamma^{n p}\right)^{\alpha}{ }_{\beta} E_{m}^{\beta}=\partial_{[n} A_{p] m}
\end{gathered}
$$

where $D_{\alpha}=\frac{\partial}{\partial \theta^{\alpha}}+\frac{i}{2} \gamma_{\alpha \beta}^{m} \theta^{\beta} \partial_{m}$ is the $\mathrm{N}=1 \mathrm{D}=10$ supersymmetric derivative. Similarly, $Q V_{s Y M}=$ $\bar{Q} V_{s Y M}=0$ implies that

$$
\begin{gathered}
\gamma_{m n p q r}^{\alpha \beta} D_{\alpha} A_{\beta I}=0, \\
A_{n I}=-\frac{i}{8} D_{\alpha} \gamma_{m}^{\alpha \beta} A_{\beta I}, \quad W_{I}^{\beta}=-\frac{i}{10} \gamma^{n \alpha \beta}\left(D_{\alpha} A_{n I}-\partial_{n} A_{\alpha I}\right), \quad U_{n p I}=\frac{1}{8} D_{\alpha}\left(\gamma_{n p}\right)^{\alpha}{ }_{\beta} W_{I}^{\beta}=\partial_{[n} A_{p] I} .
\end{gathered}
$$

Equations (20) and (22) are the linearized $N=1$ supergravity and super-Yang-Mills equations of motion written in terms of the superfields $A_{\alpha m}$ and $A_{\alpha I}$, and equations (21) and (23) define the linearized supergravity and super-Yang-Mills connections and field-strengths in terms of $A_{\alpha m}$ and $A_{\alpha I}$. For example, the on-shell graviton $h_{n m}$ and gluon $a_{n I}$ are contained in the $i\left(\gamma^{n} \theta\right)_{\alpha} h_{n m}(x)$ and $i\left(\gamma^{n} \theta\right)_{\alpha} a_{n I}(x)$ components of $A_{\alpha m}(x, \theta)$ and $A_{\alpha I}(x, \theta)$. The linearized equations of (20)-(23) will be generalized to covariant non-linear equations in the following subsections. 


\subsection{Heterotic superstring in a curved background}

The heterotic sigma model action in a curved background can be constructed by adding the massless vertex operators of (17) and (18) to the flat action of (9), and then covariantizing with respect to $N=1 D=10$ super-reparameterization invariance. Alternatively, one can consider the most general action constructed from the worldsheet variables which is classically invariant under worldsheet conformal transformations. In addition, for quantum worldsheet conformal invariance, one needs to include a Fradkin-Tseytlin term which couples the spacetime dilaton to the worldsheet curvature.

Using the worldsheet variables defined in the previous subsection, we can write the heterotic sigma model action in the form

$$
\begin{gathered}
S=\frac{1}{2 \pi \alpha^{\prime}} \int d^{2} z\left[\frac{1}{2}\left(G_{M N}(Z)+B_{M N}(Z)\right) \partial Z^{M} \bar{\partial} Z^{N}+E_{M}^{\alpha}(Z) d_{\alpha} \bar{\partial} Z^{M}+\Omega_{M \alpha}{ }^{\beta}(Z) \lambda^{\alpha} w_{\beta} \bar{\partial} Z^{M}\right. \\
\left.+A_{M I}(Z) \partial Z^{M} \bar{J}^{I}+W_{I}^{\alpha}(Z) d_{\alpha} \bar{J}^{I}+\frac{1}{2} U_{I \alpha}{ }^{\beta} \lambda^{\alpha} w_{\beta} \bar{J}^{I}+\frac{1}{2} \alpha^{\prime} \Phi(Z) r+\bar{b} \partial \bar{c}\right]+S_{\lambda}+S_{J}
\end{gathered}
$$

where $M=(m, \mu)$ are curved superspace indices, $Z^{M}=\left(x^{m}, \theta^{\mu}\right), A=(a, \alpha)$ are tangent superspace indices, $S_{\lambda}$ and $S_{J}$ are the same as in the flat action of (9), $r$ is the worldsheet curvature, and $\left[G_{M N}, B_{M N}, E_{M}^{\alpha}, \Omega_{M \alpha}{ }^{\beta}, A_{M I}, W_{I}^{\alpha}, U_{I \alpha}{ }^{\beta}, \Phi\right]$ are the background superfields. The "metric" $G_{M N}$ is defined in terms of the vectorial part of the supervielbein by $G_{M N}=$ $E_{N}{ }^{b} E_{M}{ }^{a} \eta_{a b}$, and we shall define $E_{A}{ }^{M}$ to be the inverse of $E_{M}{ }^{A}$.

Ignoring the Fradkin-Tseytlin term $\int d^{2} z \Phi(Z) r,(24)$ is the most general action with classical worldsheet conformal invariance and zero ghost number which can be constructed from the heterotic worldsheet variables. Note that $d_{\alpha}$ carries conformal weight $(1,0), \lambda^{\alpha}$ carries ghost number +1 and conformal weight $(0,0)$, and $w_{\alpha}$ carries ghost number -1 and conformal weight $(1,0)$. Since the conjugate momentum ghost variable $w_{\alpha}$ can only appear in combinations which preserve the gauge invariance $\delta w_{\alpha}=\Lambda^{a}\left(\gamma_{a} \lambda\right)_{\alpha}$, the background superfields $\Omega_{M \alpha}{ }^{\beta}$ and $U_{I \alpha}{ }^{\beta}$ must satisfy $\left(\gamma^{b c d e}\right)_{\beta}{ }^{\alpha} \Omega_{M \alpha}{ }^{\beta}=\left(\gamma^{b c d e}\right)_{\beta}{ }^{\alpha} U_{I \alpha}{ }^{\beta}=0$, i.e.

$$
\Omega_{M \alpha}{ }^{\beta}=\Omega_{M}^{(s)} \delta_{\alpha}^{\beta}+\frac{1}{2} \Omega_{M}^{c d}\left(\gamma_{c d}\right)_{\alpha}^{\beta}, \quad U_{I \alpha}{ }^{\beta}=U_{I}^{(s)} \delta_{\alpha}^{\beta}+\frac{1}{2} U_{I}^{c d}\left(\gamma_{c d}\right)_{\alpha}{ }^{\beta} .
$$

It is worthwhile to pause here to say a few words about the geometry of the target space which is implied by this action. Clearly, we identify $E_{M}{ }^{A}$ as the usual supervielbein matrix, $B_{M N}$ as the two-form potential and $\Phi$ as the dilaton. The superfield $A_{M I}$ is the super-Yang-Mills potential while the superfields $W_{I}^{\alpha}$ and $U_{I \alpha}{ }^{\beta}$ will turn out to be related to the spinor and vector super-Yang-Mills field strengths. The way in which the supervielbein enters into the action indicates that the tangent space should be a direct sum of bosonic and fermionic subspaces. This is different from the structure of the tangent space in the Green-Schwarz formalism since the $E_{M}{ }^{\alpha}$ components of the super-vielbein do not appear in the GS action. So one only needs to specify the fermionic subspace of the GS tangent space (or, dually, the bosonic subspace of the GS cotangent space). The form of the "metric" $G_{M N}=E_{N}{ }^{b} E_{M}{ }^{a} \eta_{a b}$ shows that the structure group in the bosonic sector is the Lorentz group while the existence of pure spinors implies that 
the fermionic structure group is the spin group times scale transformations. At this stage, the two Lorentz groups (in the spinor and vector sectors) are independent, although later on we shall choose a gauge with respect to one of them after which they will become identified. Note also that the spin connection $\Omega_{M \alpha}{ }^{\beta}$ appears explicitly in the action. This implies that conventional constraints corresponding to tensorial shifts of the connection are restricted by the demand that the BRST operator and action be unchanged.

Taking all this into account we find that, in addition to being invariant under target-space super-reparameterizations, the action of (24) is invariant under the local gauge transformations

$$
\begin{gathered}
\delta E_{M}^{b}=\eta_{c d} \Lambda^{b c} E_{M}^{d}, \quad \delta E_{M}^{\alpha}=\Sigma_{\beta}^{\alpha} E_{M}^{\beta}, \quad \delta \Omega_{M \alpha}{ }^{\beta}=\partial_{M} \Sigma_{\alpha}^{\beta}+\Sigma_{\alpha}^{\gamma} \Omega_{M \gamma}{ }^{\beta}-\Sigma_{\gamma}^{\beta} \Omega_{M \alpha}{ }^{\gamma} \\
\delta W_{I}^{\alpha}=\Sigma_{\alpha}^{\gamma} W_{I}^{\gamma}, \quad \delta U_{I \alpha}{ }^{\beta}=\Sigma_{\alpha}^{\gamma} U_{I \gamma}{ }^{\beta}-\Sigma_{\gamma}^{\beta} U_{I \alpha}{ }^{\gamma}, \quad \delta \lambda^{\alpha}=\Sigma_{\gamma}^{\alpha} \lambda^{\gamma}, \quad \delta w_{\alpha}=-\Sigma_{\alpha}^{\gamma} w_{\gamma}
\end{gathered}
$$

where $\Sigma_{\alpha}^{\beta}=\Sigma^{(s)} \delta_{\alpha}^{\beta}+\frac{1}{2} \Sigma^{b c}\left(\gamma_{b c}\right)_{\alpha}^{\beta}, \Lambda^{b c}$ and $\Sigma^{b c}$ parameterize independent local Lorentz transformations on the vector and spinor indices, and $\Sigma^{(s)}$ parameterizes local scale transformations on the spinor indices. Furthermore, the action of (24) and the BRST operator $\lambda^{\alpha} d_{\alpha}$ are invariant under the local shift transformations

$$
\delta \Omega_{\alpha}^{(s)}=\left(\gamma_{c}\right)_{\alpha \beta} h^{c \beta}, \quad \delta \Omega_{\alpha}^{b c}=2\left(\gamma^{[b}\right)_{\alpha \beta} h^{c] \beta}, \quad \delta d_{\alpha}=-\delta \Omega_{\alpha \beta}{ }^{\gamma} \lambda^{\beta} w_{\gamma}, \quad \delta U_{I \alpha}{ }^{\beta}=W_{I}^{\gamma} \delta \Omega_{\gamma \alpha}{ }^{\beta},
$$

where $\Omega_{\alpha \beta}^{\gamma}=E_{\alpha}^{M} \Omega_{M \beta} \gamma, h^{c \delta}$ is a local gauge parameter, and the transformation of $\Omega_{\alpha \beta} \gamma$ has been chosen such that $\lambda^{\alpha} \delta d_{\alpha}=0$. Note that $d_{\alpha}$ can be treated as an independent variable in the action of (24) since $p_{\alpha}$ does not appear explicitly.

The first term in the first and second line of (24) is the standard heterotic GS action, but the other terms will be needed for BRST invariance, just as in the linearized vertex operators of (17) and (18). As will now be shown to lowest order in $\alpha^{\prime}$, nilpotence and holomorphicity of $\lambda^{\alpha} d_{\alpha}$ implies the equations of motion for the background superfields in (24). Note that nilpotence and antiholomorphicity of the right-moving BRST current, $\bar{c} \bar{T}+\bar{c} \bar{\partial} \bar{c} \bar{b}$, does not impose any conditions to lowest order in $\alpha^{\prime}$ because the action of (24) is classically conformally invariant.

\subsection{Heterotic nilpotency constraints}

We shall first derive the constraints coming from nilpotency of $Q=\oint \lambda^{\alpha} d_{\alpha}$. Defining the canonical momentum $P_{M}$ in the usual manner as $P_{M}=\partial L / \partial\left(\partial_{0} Z^{M}\right)$, one finds that

$$
d_{\alpha}=E_{\alpha}^{M}\left[P_{M}+\frac{1}{2} B_{M N}\left(\partial Z^{N}-\bar{\partial} Z^{N}\right)-\Omega_{M \alpha}{ }^{\beta} \lambda^{\alpha} w_{\beta}-A_{M I} \bar{J}^{I}\right] .
$$

Using the canonical commutation relations

$$
\left[P_{M}, Z^{N}\right]=\delta_{M}^{N}, \quad\left[w_{\alpha}, \lambda^{\beta}\right]=\delta_{\alpha}^{\beta}, \quad\left[\bar{J}^{I}, \bar{J}^{J}\right]=f_{K}^{I J} \bar{J}^{K},
$$

one computes that

$$
\{Q, Q\}=\oint \lambda^{\alpha} \lambda^{\beta}\left[T_{\alpha \beta}{ }^{C} D_{C}+\frac{1}{2} H_{\alpha \beta M}\left(\partial Z^{M}-\bar{\partial} Z^{M}\right)-R_{\alpha \beta \gamma}{ }^{\delta} \lambda^{\gamma} w_{\delta}-F_{\alpha \beta I} \bar{J}^{I}\right]
$$


where $D_{C}=E_{C}^{M}\left(P_{M}-\Omega_{M \alpha}{ }^{\beta} \lambda^{\alpha} w_{\beta}-A_{M I} \bar{J}^{I}\right)$. The torsions $T_{A B}{ }^{C}$, three-form $H_{A B C}$, curvatures $R_{A B \gamma}{ }^{\delta}$, and field strengths $F_{A B I}$ in (30) are defined by

$$
\left[\nabla_{A}, \nabla_{B}\right]=T_{A B}{ }^{C} \nabla_{C}+R_{A B}^{(s)} S+R_{A B}^{a b} M_{a b}+F_{A B} Y^{I}, \quad H_{A B C}=3 E_{A}^{M} E_{B}^{N} E_{C}^{P} \partial_{[M} B_{N P]},
$$

where $\nabla_{A}=E_{A}^{M}\left(\partial_{M}+\Omega_{M}^{(s)} S+\Omega_{M}^{a b} M_{a b}+A_{M I} Y^{I}\right), S$ is a scale generator which transforms $\delta E_{\alpha}{ }^{M}=\Lambda^{(s)} E_{\alpha}{ }^{M}, M_{a b}$ is the Lorentz generator, $Y^{I}$ is the gauge group generator and $R_{A B \beta}{ }^{\gamma}=$ $R_{A B}^{(s)}+\frac{1}{2} R_{A B} c d\left(\gamma_{c d}\right)_{\beta} \gamma$. Note that $f_{[A B]}$ signifies the graded commutator, i.e. $f_{[A B]}=\frac{1}{2}\left(f_{A B}+\right.$ $\left.f_{B A}\right)$ when both indices are fermionic and $f_{[A B]}=\frac{1}{2}\left(f_{A B}-f_{B A}\right)$ otherwise.

So nilpotency of $Q$ implies the constraints

$$
\lambda^{\alpha} \lambda^{\beta} T_{\alpha \beta}^{C}=\lambda^{\alpha} \lambda^{\beta} H_{\alpha \beta B}=\lambda^{\alpha} \lambda^{\beta} \lambda^{\gamma} R_{\alpha \beta \gamma}{ }^{\delta}=\lambda^{\alpha} \lambda^{\beta} F_{\alpha \beta I}=0
$$

for any $\lambda^{\alpha}$ satisfying the pure spinor constraint of (2). Note that the $\lambda^{\alpha} \lambda^{\beta} \lambda^{\gamma} R_{\alpha \beta \gamma}{ }^{\delta}=0$ constraint is implied by $\lambda^{\alpha} \lambda^{\beta} T_{\alpha \beta}{ }^{C}=0$ through Bianchi identities.

As shown in [27], the constraints (32) follow from pure spinor integrability in loop superspace and imply all the essential $\mathrm{N}=1$ supergravity/super-Yang-Mills constraints. Indeed, the chirality operator introduced in [27] in pure spinor loop superspace precisely coincides with the BRST operator $Q$. So (32) implies all but the "conventional" constraints which define the vector components of superfields in terms of their spinor components and define the spin connection in terms of the super-vierbein. As will be shown below, these conventional constraints (up to gauge invariances) are implied by the holomorphicity of $\lambda^{\alpha} d_{\alpha}$.

\subsection{Heterotic holomorphicity constraints}

We shall now derive the constraints coming from holomorphicity of $\lambda^{\alpha} d_{\alpha}$. Varying $\lambda^{\alpha}$ and its conjugate momentum in (24) and ignoring the contribution from the Fradkin-Tseytlin term which is higher order in $\alpha^{\prime}$, one obtains the equations

$$
\bar{\partial} \lambda^{\alpha}=-\left(\Omega_{M \beta}{ }^{\alpha} \bar{\partial} Z^{M}+U_{I \beta}^{\alpha} \bar{J}^{I}\right) \lambda^{\beta}, \quad \bar{\partial} w_{\alpha}=\left(\Omega_{M \alpha}{ }^{\beta} \bar{\partial} Z^{M}+U_{I \alpha}{ }^{\beta} \bar{J}^{I}\right) w_{\beta},
$$

and varying the right-moving variables, one obtains the equations

$$
\partial \bar{J}^{K}=f_{J}^{I K}\left(A_{M I} \partial Z^{M}+W_{I}^{\alpha} d_{\alpha}+U_{I \alpha}^{\beta} \lambda^{\alpha} w_{\beta}\right) \bar{J}^{J}
$$

where $f_{J}^{I K}$ are the Lie algebra structure constants. And by varying $d_{\alpha}$, one obtains the equation of motion

$$
E_{M}^{\alpha} \bar{\partial} Z^{M}=-W_{I}^{\alpha} \bar{J}^{I}
$$

Finally, by computing $E_{\alpha}^{P}\left(\delta S / \delta Z^{P}\right)$, one obtains the equation of motion

$$
\begin{aligned}
& \bar{\partial} d_{\alpha}=E_{\alpha}^{P}\left[\left(\partial_{[P} E_{M]}^{a} E_{N}^{b} \eta_{a b}+\partial_{[P} E_{N]}^{a} E_{M}^{b} \eta_{a b}+\frac{1}{2} H_{P M N}\right) \partial Z^{M} \bar{\partial} Z^{N}\right. \\
& +2\left(\partial_{[P} E_{N]}^{\beta} d_{\beta}+\partial_{[P} \Omega_{N] \gamma}{ }^{\beta} \lambda^{\gamma} w_{\beta}\right) \bar{\partial} Z^{N}-\Omega_{P \gamma}{ }^{\beta} \bar{\partial}\left(\lambda^{\gamma} w_{\beta}\right)-A_{P I} \partial \bar{J}^{I} \\
& \left.+\left(2 \partial_{[P} A_{M] I} \partial Z^{M}+\partial_{P} W_{I}^{\beta} d_{\beta}+\partial_{P} U_{I \gamma}{ }^{\beta} \lambda^{\gamma} w_{\beta}\right) \bar{J}^{I}\right] .
\end{aligned}
$$


Putting these equations together, one finds

$$
\begin{aligned}
\bar{\partial}\left(\lambda^{\alpha} d_{\alpha}\right)=\lambda^{\alpha} & \left.\frac{1}{2}\left(T_{\alpha b c}+T_{\alpha c b}+H_{\alpha b c}\right) \Pi^{b}+\frac{1}{2}\left(T_{\alpha \beta c}+H_{\alpha \beta c}\right) \Pi^{\beta}+T_{\alpha c}{ }^{\beta} d_{\beta}+R_{\alpha c \beta}{ }^{\gamma} \lambda^{\beta} w_{\gamma}\right] \bar{\Pi}^{c} \\
& +\lambda^{\alpha}\left[\left(F_{\alpha b I}-\frac{1}{2} W_{I}^{\beta}\left(T_{\alpha \beta b}+H_{\alpha b \beta}\right)\right) \Pi^{b}+\left(F_{\alpha \gamma I}-\frac{1}{2} W_{I}^{\beta} H_{\alpha \gamma \beta}\right) \Pi^{\gamma}\right] \bar{J}^{I} \\
& +\lambda^{\alpha}\left[\left(\nabla_{\alpha} W_{I}^{\beta}-T_{\alpha \gamma}{ }^{\beta} W_{I}^{\gamma}-U_{I \alpha}{ }^{\beta}\right) d_{\beta}+\left(\nabla_{\alpha} U_{\gamma I}^{\delta}-R_{\alpha \beta \gamma}{ }^{\delta} W_{I}^{\beta}\right) \lambda^{\gamma} w_{\delta}\right] \bar{J}^{I},
\end{aligned}
$$

where $\Pi^{A}=E_{M}^{A} \partial Z^{M}, \bar{\Pi}^{A}=E_{M}^{A} \bar{\partial} Z^{M}$ and $T_{A B c}=T_{A B}{ }^{d} \eta_{c d}$.

So from (37), $\bar{\partial}\left(\lambda^{\alpha} d_{\alpha}\right)=0$ implies the constraints

$$
\begin{gathered}
T_{\alpha(a b)}=H_{\alpha a b}=T_{\alpha \beta c}+H_{\alpha \beta c}=T_{\alpha c}{ }^{\beta}=0, \quad \lambda^{\alpha} \lambda^{\beta} R_{\alpha c \beta}{ }^{\gamma}=0, \quad F_{\alpha \beta I}=\frac{1}{2} W_{I}^{\gamma} H_{\alpha \beta \gamma}, \\
F_{\alpha b I}=W_{I}^{\beta} T_{\alpha \beta b}, \quad \nabla_{\alpha} W_{I}^{\beta}-T_{\alpha \gamma}{ }^{\beta} W_{I}^{\gamma}=U_{I \alpha}{ }^{\beta}, \quad \lambda^{\alpha} \lambda^{\beta}\left(\nabla_{\alpha} U_{I \beta}{ }^{\delta}-R_{\alpha \gamma \beta}{ }^{\delta} W_{I}^{\gamma}\right)=0,
\end{gathered}
$$

where $\lambda^{\alpha}$ is any spinor satisfying (2).

The constraints of (32) and (38) will now be shown to imply the correct supergravity and super-Yang-Mills equations of motion.

\section{5 $\mathrm{N}=1$ supergravity/super-Yang-Mills constraints}

It will be useful to first consider the supergravity constraints of (32) and (38) which have lowest scaling dimension since the higher dimensional constraints will be implied by these constraints through Bianchi identities. At dimension $-\frac{1}{2}$, the only constraint is $\lambda^{\alpha} \lambda^{\beta} H_{\alpha \beta \gamma}=0$ which implies that $H_{\alpha \beta \gamma}=0$ since there is no non-zero symmetric $H_{\alpha \beta \gamma}$ satisfying $\lambda^{\alpha} \lambda^{\beta} H_{\alpha \beta \gamma}=0$.

At dimension 0 , the constraints $\lambda^{\alpha} \lambda^{\beta} T_{\alpha \beta}{ }^{c}=\lambda^{\alpha} \lambda^{\beta} H_{\alpha \beta c}=0$ and $T_{\alpha \beta}{ }^{c}=-\eta^{c d} H_{\alpha \beta d}$ imply that $T_{\alpha \beta}^{c}=-\eta^{c d} H_{\alpha \beta d}=i\left(\gamma^{d}\right)_{\alpha \beta} f_{d}^{c}$ for some $f_{d}^{c}$. The dimension zero Bianchi identity $D_{(\alpha} H_{\beta \gamma \delta)}=T_{(\alpha \beta}^{D} H_{\gamma \delta) D}$ then tells us that $f_{d}^{c}$ is an $\mathrm{SO}(9,1)$ matrix times a scale factor. So using the local spinor Lorentz and scale transformations of $(26), f_{d}^{c}$ can be gauge-fixed to $\delta_{d}^{c}$ so that

$$
T_{\alpha \beta}^{c}=-\eta^{c d} H_{\alpha \beta d}=i\left(\gamma^{c}\right)_{\alpha \beta} .
$$

Note that at this point we still have one local Lorentz symmetry, acting now on both spinor and vector indices. The connection for this symmetry is $\Omega_{M}{ }^{a b}$. On the other hand, the fermionic scale invariance has been fixed and so it need not be the case that other components of the torsion should respect this symmetry.

At dimension $\frac{1}{2}$, the constraint $\lambda^{\alpha} \lambda^{\beta} T_{\alpha \beta} \gamma=0$ implies that $T_{\alpha \beta} \gamma=f_{c}^{\gamma}\left(\gamma^{c}\right)_{\alpha \beta}$ for some $f_{c}^{\gamma}$. Using the shift symmetry of $(27), f_{c}^{\gamma}$ can be gauge-fixed to zero so that $T_{\alpha \beta} \gamma=0$. The other

dimension $\frac{1}{2}$ constraints, $H_{\alpha c d}=T_{\alpha(c d)}=0$, imply through the Bianchi identity $\nabla_{(\alpha} T_{\beta \gamma)}{ }^{c}=$ $-T_{(\alpha \beta}{ }^{D} T_{\gamma) D}{ }^{c}$ that $T_{\gamma b}^{c}=2 \eta^{c d}\left(\gamma_{b d}\right)_{\alpha}^{\beta} \Omega_{\beta}^{(s)}$.

At dimension one, the constraint $T_{c \alpha}{ }^{\beta}=0$ decomposes into

$$
T_{c \alpha}^{\beta}=T_{c}^{\text {defg }}\left(\gamma_{\text {defg }}\right)_{\alpha}^{\beta}+T_{c}^{\text {de }}\left(\gamma_{d e}\right)_{\alpha}^{\beta}+T_{c} \delta_{\alpha}^{\beta}=0 .
$$


The constraints $T_{c}=0$ and $T_{c}^{d e}=0$ determine the vector components of the spin connections $\Omega_{c}^{(s)}$ and $\Omega_{c}^{d e}$, whereas the constraint $T_{c}^{d e f g}=0$ is implied by the Bianchi identity $(\nabla H+$ $T H)_{b c \alpha \gamma}\left(\gamma^{b d e f g}\right)^{\alpha \gamma}=0$. Similarly, the constraints involving the curvature tensor in (32) and (38) are implied by the Bianchi identity $R_{[A B C]}{ }^{D}=\nabla_{[A} T_{B C]}^{D}+T_{[A B}{ }^{E} T_{C] E}{ }^{F}$.

To extract the supergravity equations unambiguously from the above constraints it is convenient to reduce the structure group from Lorentz group times fermionic scale to just the Lorentz group. The dimension zero torsions are unchanged but the dimension one-half torsion $T_{\alpha \beta}{ }^{\gamma}$ gets amended to

$$
T_{\alpha \beta}^{\gamma} \rightarrow T_{\alpha \beta}^{\gamma}-2 \delta_{(\alpha}^{\gamma} \Omega_{\beta)}^{(s)}=-2 \delta_{(\alpha}^{\gamma} \Omega_{\beta)}^{(s)}
$$

There are corresponding changes at higher dimensions. The leading component of $\Omega_{\alpha}^{(s)}$ is the dilatino and to show that there are no unwanted fields one must show that this superfield is proportional to the spinorial derivative of a scalar superfield $\Phi$ whose leading component is the dilaton. It is straightforward to verify that this is the case, although it is necessary to go to dimension three-halves to do so. As discussed in section 5, holomorphicity of $\lambda^{\alpha} d_{\alpha}$ to the next order in $\alpha^{\prime}$ will imply that this scalar superfield $\Phi$ is the same superfield that appears in the Fradkin-Tseytlin term of (24).

The above supergravity constraints therefore imply that all of the supergravity superfields can be expressed in terms of the spinor supervielbein $E_{\alpha}{ }^{M}$, and the equation $T_{\alpha \beta}{ }^{c}=i\left(\gamma^{c}\right)_{\alpha \beta}$ puts $E_{\alpha}^{M}$ on-shell. Similarly, the super-Yang-Mills constraints in (32) and (38) imply that the super-Yang-Mills superfields $A_{c I}, W_{I}^{\alpha}$ and $U_{I \alpha}{ }^{\beta}$ can be expressed in terms of the spinor superfield $A_{\alpha I}$, and the equation $F_{\alpha \beta I}=0$ puts $A_{\alpha I}$ on-shell. So nilpotence and holomorphicity of $\lambda^{\alpha} d_{\alpha}$ has been shown to imply the $\mathrm{N}=1$ supergravity/super-Yang-Mills equations of motion to lowest order in $\alpha^{\prime}$.

\section{$3 \quad$ Type II Superstring Sigma Model}

In this section, the pure spinor version of the Type IIA and IIB superstring will be reviewed in flat and curved backgrounds. Nilpotence and holomorphicity of $\lambda^{\alpha} d_{\alpha}$ and nilpotence and antiholomorphicity of $\hat{\lambda}^{\hat{\alpha}} d_{\hat{\alpha}}$ will then be shown to imply the superspace equations of motion for the $\mathrm{N}=2$ supergravity background.

\subsection{Type II superstring in a flat background}

In the pure spinor version of the Type II superstring, the worldsheet variables consist of the $N=2 D=10$ superspace variables $\left(x^{m}, \theta^{\alpha}, p_{\alpha}, \hat{\theta}^{\hat{\alpha}}, \hat{p}_{\hat{\alpha}}\right)$ for $m=0$ to 9 and $\alpha, \hat{\alpha}=1$ to 16 where $p_{\alpha}$ is the conjugate momentum to $\theta^{\alpha}$ and $\hat{p}_{\hat{\alpha}}$ is the conjugate momentum to $\hat{\theta}^{\hat{\alpha}}$. For the Type IIA superstring, $\alpha$ and $\hat{\alpha}$ denote $\mathrm{SO}(9,1)$ spinors of opposite chirality while for the Type IIB superstring, $\alpha$ and $\hat{\alpha}$ denote $\mathrm{SO}(9,1)$ spinors of the same chirality. The pure spinor formalism also contains the worldsheet variables $\lambda^{\alpha}$ and $\hat{\lambda}^{\hat{\alpha}}$, and their conjugate momenta $w_{\alpha}$ and $\hat{w}_{\hat{\alpha}}$, 
which are constrained to satisfy the pure spinor conditions

$$
\lambda \gamma^{m} \lambda=0, \quad \hat{\lambda} \gamma^{m} \hat{\lambda}=0
$$

for $m=0$ to 9 . In a flat background, $\theta^{\alpha}, p_{\alpha}, \lambda^{\alpha}$ and $w_{\alpha}$ are left-moving while $\hat{\theta}^{\hat{\alpha}}, \hat{p}_{\hat{\alpha}}, \hat{\lambda}_{\hat{\alpha}}$ and $\hat{w}_{\hat{\alpha}}$ are right-moving.

The action and stress-tensor in a flat background is

$$
\begin{gathered}
S=\frac{1}{2 \pi \alpha^{\prime}} \int d^{2} z\left(\frac{1}{2} \partial x^{m} \bar{\partial} x_{m}+p_{\alpha} \bar{\partial} \theta^{\alpha}+\hat{p}_{\hat{\alpha}} \partial \hat{\theta}^{\hat{\alpha}}\right)+S_{\lambda}+S_{\hat{\lambda}}, \\
T=\frac{1}{\alpha^{\prime}}\left(-\frac{1}{2} \partial x^{m} \partial x_{m}-p_{\alpha} \partial \theta^{\alpha}\right)+T_{\lambda}, \quad \bar{T}=\frac{1}{\alpha^{\prime}}\left(-\frac{1}{2} \bar{\partial} x^{m} \bar{\partial} x_{m}-\hat{p}_{\hat{\alpha}} \bar{\partial} \hat{\theta}^{\hat{\alpha}}\right)+\bar{T}_{\hat{\lambda}},
\end{gathered}
$$

where $S_{\lambda}$ and $S_{\hat{\lambda}}$ are the actions for $\lambda^{\alpha}$ and $\hat{\lambda}^{\hat{\alpha}}$, and $T_{\lambda}$ and $\bar{T}_{\hat{\lambda}}$ are the $c=22$ left and rightmoving stress tensors for $\lambda^{\alpha}$ and $\hat{\lambda}^{\hat{\alpha}}$. As in the heterotic case, the explicit form of $S_{\lambda}$ and $S_{\hat{\lambda}}$ will not be needed. We will only need to know that one can construct left and right-moving Lorentz currents, $N^{m n}=\frac{1}{2 \alpha^{\prime}} \lambda \gamma^{m n} w$ and $\hat{N}^{m n}=\frac{1}{2 \alpha^{\prime}} \hat{\lambda} \gamma^{m n} \hat{w}$, which satisfy the OPE's

$$
\begin{gathered}
N^{m n}(y) \lambda^{\alpha}(z) \rightarrow \frac{1}{2}\left(\gamma^{m n}\right)^{\alpha}{ }_{\beta} \frac{\lambda^{\beta}(z)}{y-z}, \quad \hat{N}^{m n}(\bar{y}) \hat{\lambda}^{\hat{\alpha}}(\bar{z}) \rightarrow \frac{1}{2}\left(\gamma^{m n}\right)^{\hat{\alpha}}{ }_{\hat{\beta}} \frac{\hat{\lambda}(\bar{z})}{\bar{y}-\bar{z}} \\
N^{k l}(y) N^{m n}(z) \rightarrow \frac{\eta^{m[l} N^{k] n}(z)-\eta^{n[l} N^{k] m}(z)}{y-z}-3 \frac{\eta^{k n} \eta^{l m}-\eta^{k m} \eta^{l n}}{(y-z)^{2}} \\
\hat{N}^{k l}(\bar{y}) \hat{N}^{m n}(\bar{z}) \rightarrow \frac{\eta^{m[l} \hat{N}^{k] n}(\bar{z})-\eta^{n[l} \hat{N}^{k] m}(\bar{z})}{\bar{y}-\bar{z}}-3 \frac{\eta^{k n} \eta^{l m}-\eta^{k m} \eta^{l n}}{(\bar{y}-\bar{z})^{2}}
\end{gathered}
$$

Physical states of the superstring are defined as vertex operators in the cohomology of the left and right-moving BRST operators

$$
Q=\oint \lambda^{\alpha} d_{\alpha}, \quad \bar{Q}=\oint \hat{\lambda}^{\hat{\alpha}} \hat{d}_{\hat{\alpha}}
$$

where

$$
\begin{array}{ll}
d_{\alpha}=p_{\alpha}-\frac{i}{2} \gamma_{\alpha \beta}^{m} \theta^{\beta} \partial x_{m}+\frac{1}{8} \gamma_{\alpha \beta}^{m} \gamma_{m} \gamma \delta \theta^{\beta} \theta^{\gamma} \partial \theta^{\delta}, & \Pi^{m}=\partial x^{m}+\frac{i}{2} \theta \gamma^{m} \partial \theta \\
\hat{d}_{\hat{\alpha}}=\hat{p}_{\hat{\alpha}}-\frac{i}{2} \gamma_{\hat{\alpha} \hat{\beta}}^{m} \hat{\theta}^{\beta} \bar{\partial} x_{m}+\frac{1}{8} \gamma_{\hat{\alpha} \hat{\beta}}^{m} \gamma_{m} \hat{\gamma} \hat{\delta} \hat{\theta}^{\hat{\beta}} \hat{\theta}^{\hat{\gamma}} \bar{\partial} \hat{\theta}^{\hat{\delta}}, & \bar{\Pi}^{m}=\bar{\partial} x^{m}+\frac{i}{2} \hat{\theta} \gamma^{m} \bar{\partial} \hat{\theta}
\end{array}
$$

are spacetime supersymmetric and satisfy the OPE's

$$
\begin{array}{ll}
d_{\alpha}(y) d_{\beta}(z) \rightarrow-i \alpha^{\prime}(y-z)^{-1} \gamma_{\alpha \beta}^{m} \Pi_{m}(z), & d_{\alpha}(y) \Pi^{m}(z) \rightarrow i \alpha^{\prime}(y-z)^{-1} \gamma_{\alpha \beta}^{m} \partial \theta^{\beta}(z), \\
\hat{d}_{\hat{\alpha}}(\bar{y}) \hat{d}_{\hat{\beta}}(\bar{z}) \rightarrow-i \alpha^{\prime}(\bar{y}-\bar{z})^{-1} \gamma_{\hat{\alpha} \hat{\beta}}^{m} \bar{\Pi}_{m}(\bar{z}), & \hat{d}_{\hat{\alpha}}(\bar{y}) \bar{\Pi}^{m}(\bar{z}) \rightarrow i \alpha^{\prime}(\bar{y}-\bar{z})^{-1} \gamma_{\hat{\alpha} \hat{\beta}}^{m} \bar{\partial} \hat{\theta}^{\hat{\beta}}(\bar{z}),
\end{array}
$$

To construct the sigma model for the Type II superstring, it will be useful to know the integrated form of the massless Type II supergravity vertex operator

$$
V_{S G}=\int d^{2} z\left[\partial \theta^{\alpha} \bar{\partial} \hat{\theta}^{\hat{\beta}} A_{\alpha \hat{\beta}}(x, \theta, \hat{\theta})+\partial \theta^{\alpha} \bar{\Pi}^{m} A_{\alpha m}(x, \theta, \hat{\theta})+\Pi^{m} \bar{\partial} \hat{\theta}^{\hat{\alpha}} A_{m \hat{\alpha}}(x, \theta, \hat{\theta})+\Pi^{m} \bar{\Pi}^{n} A_{m n}(x, \theta, \hat{\theta})\right.
$$




$$
\begin{gathered}
+d_{\alpha}\left(\bar{\partial} \hat{\theta}^{\hat{\beta}} E_{\hat{\beta}}^{\alpha}(x, \theta, \hat{\theta})+\bar{\Pi}^{m} E_{m}^{\alpha}(x, \theta, \hat{\theta})\right)+\hat{d}_{\hat{\alpha}}\left(\partial \theta^{\beta} E_{\beta}^{\hat{\alpha}}(x, \theta, \hat{\theta})+\Pi^{m} E_{m}^{\hat{\alpha}}(x, \theta, \hat{\theta})\right) \\
+\frac{1}{2} N_{m n}\left(\bar{\partial} \hat{\theta}^{\hat{\beta}} \Omega_{\hat{\beta}}^{m n}(x, \theta, \hat{\theta})+\bar{\Pi}^{p} \Omega_{p}^{m n}(x, \theta, \hat{\theta})\right)+\frac{1}{2} \hat{N}_{m n}\left(\partial \theta^{\beta} \hat{\Omega}_{\beta}^{m n}(x, \theta, \hat{\theta})+\Pi^{p} \hat{\Omega}_{p}^{m n}(x, \theta, \hat{\theta})\right) \\
\left.+d_{\alpha} \hat{d}_{\hat{\beta}} P^{\alpha \hat{\beta}}(x, \theta, \hat{\theta})+N_{m n} \hat{d}_{\hat{\alpha}} C^{m n \hat{\alpha}}(x, \theta, \hat{\theta})+d_{\alpha} \hat{N}_{m n} \hat{C}^{\alpha m n}(x, \theta, \hat{\theta})+N_{m n} \hat{N}_{p q} S^{m n p q}(x, \theta, \hat{\theta})\right] .
\end{gathered}
$$

Note that the first line of $V_{S G}$ is the same as in the Green-Schwarz Type II superstring vertex operator, but the other lines are needed for the vertex operator to be BRST invariant. The Type II superstring vertex operator of (53) can be understood as the "square" of the open superstring vertex operator of (19).

Using (42) and the OPE's of (45) and (51), one can check that $Q V_{S G}=\bar{Q} V_{S G}=0$ implies that

$$
\begin{aligned}
& \gamma_{m n p q r}^{\alpha \beta} D_{\alpha} A_{\beta \hat{\gamma}}=0, \quad \gamma_{m n p q r}^{\hat{\alpha} \hat{\beta}} \hat{D}_{\hat{\alpha}} A_{\gamma \hat{\beta}}=0 \\
& A_{n \hat{\gamma}}=-\frac{i}{8} D_{\alpha} \gamma_{n}^{\alpha \beta} A_{\beta \hat{\gamma}}, \quad A_{\gamma n}=-\frac{i}{8} \hat{D}_{\hat{\alpha}} \gamma_{n}^{\hat{\alpha} \hat{\beta}} A_{\gamma \hat{\beta}}, \quad A_{m n}=\frac{1}{64} D_{\alpha} \hat{D}_{\hat{\gamma}} \gamma_{m}^{\alpha \beta} \gamma_{n}^{\hat{\gamma} \hat{\delta}} A_{\beta \hat{\delta}},
\end{aligned}
$$

and similar equations for the superfields $(E, \Omega, \hat{\Omega}, P, C, \hat{C}, S)$ in terms of $A_{\alpha \hat{\beta}}$. Note that $D_{\alpha}=$ $\frac{\partial}{\partial \theta^{\alpha}}+\frac{i}{2} \gamma_{\alpha \beta}^{m} \theta^{\beta} \partial_{m}$ and $\hat{D}_{\hat{\alpha}}=\frac{\partial}{\partial \hat{\theta}^{\hat{\alpha}}}+\frac{i}{2} \gamma_{\hat{\alpha} \hat{\beta}}^{m} \hat{\theta}^{\hat{\beta}} \partial_{m}$ are the $\mathrm{N}=2 \mathrm{D}=10$ supersymmetric derivatives. Equations (54) are the linearized $N=2$ supergravity equations of motion written in terms of the superfield $A_{\alpha \hat{\beta}}$, and equations (55) define the linearized supergravity connections in terms of $A_{\alpha \hat{\beta}}$. For example, the on-shell graviton $h_{n m}$ is contained in the $\left(\gamma^{n} \theta\right)_{\alpha}\left(\gamma^{m} \hat{\theta}\right)_{\hat{\beta}} h_{n m}(x)$ of $A_{\alpha \hat{\beta}}(x, \theta, \hat{\theta})$. These linearized equations will be generalized to covariant non-linear equations in the following subsections.

\subsection{Type II superstring in a curved background}

The Type II sigma model action in a curved background (except for the Fradkin-Tseytlin term) can be constructed by adding the massless vertex operator of (53) to the flat action of (43), and then covariantizing with respect to $N=2 D=10$ super-reparameterization invariance. Alternatively, one can consider the most general action constructed from the worldsheet variables which is classically invariant under worldsheet conformal transformations.

Using the worldsheet variables defined in the previous subsection, we can write the Type II sigma model action as

$$
\begin{gathered}
S=\frac{1}{2 \pi \alpha^{\prime}} \int d^{2} z\left[\frac{1}{2}\left(G_{M N}(Z)+B_{M N}(Z)\right) \partial Z^{M} \bar{\partial} Z^{N}\right. \\
+E_{M}^{\alpha}(Z) d_{\alpha} \bar{\partial} Z^{M}+E_{M}^{\hat{\alpha}}(Z) \hat{d}_{\hat{\alpha}} \partial Z^{M}+\Omega_{M \alpha}{ }^{\beta}(Z) \lambda^{\alpha} w_{\beta} \bar{\partial} Z^{M}+\hat{\Omega}_{M \hat{\alpha}} \hat{\beta}^{\hat{\alpha}}(Z) \hat{\lambda}^{\hat{\alpha}} \hat{w}_{\hat{\beta}} \partial Z^{M}+P^{\alpha \hat{\beta}}(Z) d_{\alpha} \hat{d}_{\hat{\beta}} \\
\left.+C_{\alpha}^{\beta \hat{\gamma}}(Z) \lambda^{\alpha} w_{\beta} \hat{d}_{\hat{\gamma}}+\hat{C}_{\hat{\alpha}}^{\hat{\beta} \gamma}(Z) \hat{\lambda}^{\hat{\alpha}} \hat{w}_{\hat{\beta}} d_{\gamma}+S_{\alpha \hat{\gamma}}^{\beta \hat{\delta}}(Z) \lambda^{\alpha} w_{\beta} \hat{\lambda}^{\hat{\gamma}} \hat{w}_{\hat{\delta}}+\frac{1}{2} \alpha^{\prime} \Phi(Z) r\right]+S_{\lambda}+S_{\hat{\lambda}}
\end{gathered}
$$

where $M=(m, \mu, \hat{\mu})$ are curved superspace indices, $Z^{M}=\left(x^{m}, \theta^{\mu}, \hat{\theta}^{\hat{\mu}}\right), A=(a, \alpha, \hat{\alpha})$ are tangent superspace indices, $S_{\lambda}$ and $S_{\hat{\lambda}}$ are the same as in the flat action of (43), $r$ is the worldsheet 
curvature, and $\left[G_{M N}=\eta_{c d} E_{M}^{c} E_{N}^{d}, B_{M N}, E_{M}^{\alpha}, E_{M}^{\hat{\alpha}}, \Omega_{M \alpha}^{\beta}, \hat{\Omega}_{M \hat{\alpha}}^{\hat{\beta}}, P^{\alpha \hat{\beta}}, C_{\alpha}^{\beta \hat{\gamma}}, \hat{C}_{\hat{\alpha}}^{\hat{\beta} \gamma}, S_{\alpha \hat{\gamma}}^{\beta \hat{\delta}}, \Phi\right]$ are the background superfields.

If the Fradkin-Tseytlin term, $\int d^{2} z \Phi(Z) r$, is omitted (56) is the most general action with classical worldsheet conformal invariance and zero (left,right)-moving ghost number which can be constructed from the Type II worldsheet variables. Note that $d_{\alpha}$ carries conformal weight $(1,0), \hat{d}_{\hat{\alpha}}$ carries conformal weight $(0,1), \lambda^{\alpha}$ carries ghost number $(1,0)$ and conformal weight $(0,0), \hat{\lambda}^{\hat{\alpha}}$ carries ghost number $(0,1)$ and conformal weight $(0,0), w_{\alpha}$ carries ghost number $(-1,0)$ and conformal weight $(1,0)$, and $\hat{w}_{\hat{\alpha}}$ carries ghost number $(0,-1)$ and conformal weight $(0,1)$. Since $w_{\alpha}$ and $\hat{w}_{\hat{\alpha}}$ can only appear in combinations which commute with the pure spinor constraints of (42), the background superfields must satisfy

$$
\left(\gamma^{b c d e}\right)_{\beta}^{\alpha} \Omega_{M \alpha}^{\beta}=\left(\gamma^{b c d e}\right)_{\beta}^{\alpha} \hat{\Omega}_{M \hat{\alpha}}^{\hat{\beta}}=\left(\gamma^{b c d e}\right)_{\beta}^{\alpha} C_{\alpha}^{\beta \hat{\gamma}}=\left(\gamma^{b c d e}\right)_{\hat{\beta}}^{\hat{\alpha}} \hat{C}_{\hat{\alpha}}^{\hat{\beta} \gamma}=\left(\gamma^{b c d e}\right)_{\beta}^{\alpha} S_{\alpha \hat{\gamma}}^{\beta \hat{\delta}}=\left(\gamma^{b c d e}\right)_{\hat{\delta}}^{\hat{\gamma}} S_{\alpha \hat{\gamma}}^{\beta \hat{\delta}}=0
$$

and the different components of the spin connections will be defined as

$$
\Omega_{M \alpha}{ }^{\beta}=\Omega_{M}^{(s)} \delta_{\alpha}^{\beta}+\frac{1}{2} \Omega_{M}^{c d}\left(\gamma_{c d}\right)_{\alpha}^{\beta}, \quad \hat{\Omega}_{M \hat{\alpha}}^{\hat{\beta}}=\hat{\Omega}_{M}^{(s)} \delta_{\hat{\alpha}}^{\hat{\beta}}+\frac{1}{2} \hat{\Omega}_{M}^{c d}\left(\gamma_{c d}\right)_{\hat{\alpha}}^{\hat{\beta}} .
$$

Although the background superfields appearing in (56) look unconventional, they all have physical interpretations. The superfields $E_{M}{ }^{A}, B_{M N}$ and $\Phi$ are the supervielbein, two-form potential and dilaton superfields, $P^{\alpha \hat{\beta}}$ is the superfield whose lowest components are the Type II Ramond-Ramond field strengths, and the superfields $C_{\alpha}^{\beta \hat{\gamma}}=C^{\hat{\gamma}} \delta_{\alpha}^{\beta}+\frac{1}{2} C^{\hat{\gamma} a b}\left(\gamma_{a b}\right)_{\alpha}^{\beta}$ and $\hat{C}_{\hat{\alpha}}^{\hat{\beta} \gamma}=$ $\hat{C}^{\gamma} \delta_{\hat{\alpha}}^{\hat{\beta}}+\frac{1}{2} \hat{C}^{\gamma a b}\left(\gamma_{a b}\right)_{\hat{\alpha}}^{\hat{\beta}}$ are related to the $\mathrm{N}=2 \mathrm{D}=10$ dilatino and gravitino field strengths. As in the heterotic sigma model, the form of the metric in the Type II sigma model implies that the structure group in the bosonic sector is the Lorentz group. But there are now two independent pure spinors, so one has two independent fermionic structure groups, each consisting of the spin group times scale transformations. One therefore has two independent sets of spin connections and scale connections, $\left(\Omega_{M}^{(s)}, \Omega_{M}^{a b}\right)$ and $\left(\hat{\Omega}_{M}^{(s)}, \hat{\Omega}_{M}^{a b}\right)$, which appear explicitly in the Type II sigma model action. Finally, the background superfields $S_{\alpha \hat{\gamma}}^{\beta \hat{\delta}}$ appearing in (56) will be related to curvatures constructed from these spin and scale connections. Note that a similar relation occurs in the RNS sigma model action which contains the terms

$$
\frac{1}{4 \pi \alpha^{\prime}} \int d^{2} z\left(\Omega_{m}^{a b}(x) \psi_{a} \psi_{b} \bar{\partial} x^{m}+\hat{\Omega}_{m}^{a b}(x) \bar{\psi}_{a} \bar{\psi}_{b} \partial x^{m}+S_{a b c d}(x) \psi^{a} \psi^{b} \bar{\psi}^{c} \bar{\psi}^{d}\right)
$$

where $\psi^{a}=e_{m}^{a}(x) \psi^{m}, \bar{\psi}^{a}=e_{m}^{a}(x) \bar{\psi}^{m}$, and $e_{m}^{a}(x)$ is the target-space vielbein.

In addition to being target-space super-reparameterization invariant, the action of (56) is invariant under the local gauge transformations

$$
\begin{gathered}
\delta E_{M}^{b}=\eta_{c d} \Lambda^{b c} E_{M}^{d}, \quad \delta E_{M}^{\alpha}=\Sigma_{\beta}^{\alpha} E_{M}^{\beta}, \quad \delta E_{M}^{\hat{\alpha}}=\hat{\Sigma}_{\hat{\beta}}^{\hat{\alpha}} E_{M}^{\hat{\beta}}, \\
\delta \Omega_{M \alpha}^{\beta}=\partial_{M} \Sigma_{\alpha}^{\beta}+\Sigma_{\alpha}^{\gamma} \Omega_{M \gamma}{ }^{\beta}-\Sigma_{\gamma}^{\beta} \Omega_{M \alpha}^{\gamma}, \quad \delta \hat{\Omega}_{M \hat{\alpha}}^{\hat{\beta}}=\partial_{M} \hat{\Sigma}_{\hat{\alpha}}^{\hat{\beta}}+\hat{\Sigma}_{\hat{\alpha}}^{\hat{\gamma}} \hat{\Omega}_{M \hat{\gamma}}^{\hat{\beta}}-\hat{\Sigma}_{\hat{\gamma}}^{\hat{\beta}} \hat{\Omega}_{M \hat{\alpha}} \hat{\gamma}^{\hat{\gamma}}, \\
\delta \lambda^{\alpha}=\Sigma_{\gamma}^{\alpha} \lambda^{\gamma}, \quad \delta w_{\alpha}=-\Sigma_{\alpha}^{\gamma} w_{\gamma}, \quad \delta \hat{\lambda}^{\hat{\alpha}}=\hat{\Sigma}_{\hat{\gamma}}^{\hat{\alpha}} \hat{\lambda}^{\hat{\gamma}}, \quad \delta \hat{w}_{\hat{\alpha}}=-\hat{\Sigma}_{\hat{\alpha}}^{\hat{\gamma}} w_{\hat{\gamma}},
\end{gathered}
$$


where $\Sigma_{\alpha}^{\beta}=\Sigma^{(s)} \delta_{\alpha}^{\beta}+\frac{1}{2} \Sigma^{b c}\left(\gamma_{b c}\right)_{\alpha}^{\beta}, \hat{\Sigma}_{\hat{\alpha}}^{\hat{\beta}}=\hat{\Sigma}^{(s)} \delta_{\hat{\alpha}}^{\hat{\beta}}+\frac{1}{2} \hat{\Sigma}^{b c}\left(\gamma_{b c}\right)_{\hat{\alpha}}^{\hat{\beta}},\left[\Lambda^{b c}, \Sigma^{b c}, \hat{\Sigma}^{b c}\right]$ parameterize independent local Lorentz transformations on the [vector, unhatted spinor, hatted spinor] indices, $\Sigma^{(s)}$ and $\hat{\Sigma}^{(s)}$ parameterize independent local scale transformations on the unhatted and hatted spinor indices, and the background superfields $\left[P^{\alpha \hat{\alpha}}, C_{\alpha}^{\beta \hat{\gamma}}, \hat{C}_{\hat{\alpha}}^{\hat{\beta} \gamma}, S_{\alpha \hat{\gamma}}^{\beta \hat{\delta}}\right]$ transform according to their spinor indices.

Furthermore, the action of (56) and the BRST operators $\lambda^{\alpha} d_{\alpha}$ and $\hat{\lambda}^{\hat{\alpha}} \hat{d}_{\hat{\alpha}}$ are invariant under the local shift transformations

$$
\begin{gathered}
\delta \Omega_{\alpha}^{(s)}=\left(\gamma_{c}\right)_{\alpha \beta} h^{c \delta}, \quad \delta \Omega_{\alpha}^{b c}=2\left(\gamma^{[b}\right)_{\beta \delta} h^{c] \delta}, \quad \delta d_{\alpha}=-\delta \Omega_{\alpha \beta}{ }^{\gamma} \lambda^{\beta} w_{\gamma}, \\
\delta \hat{\Omega}_{\hat{\alpha}}^{(s)}=\left(\gamma_{c}\right)_{\hat{\alpha} \hat{\beta}} \hat{h}^{c \hat{\delta}}, \quad \delta \hat{\Omega}_{\hat{\alpha}}^{b c}=2\left(\gamma^{[b}\right)_{\hat{\beta} \hat{\delta}} \hat{h}^{c] \delta}, \quad \delta \hat{d}_{\alpha}=-\delta \hat{\Omega}_{\hat{\alpha} \hat{\beta}} \hat{\gamma}^{\hat{\beta}} \hat{w}_{\hat{\gamma}}, \\
\delta C_{\alpha}^{\beta \hat{\gamma}}=P^{\delta \hat{\gamma}} \delta \Omega_{\delta \alpha}{ }^{\beta}, \quad \delta \hat{C}_{\hat{\alpha}}^{\hat{\beta} \gamma}=-P^{\gamma \hat{\delta}} \delta \hat{\Omega}_{\hat{\delta} \hat{\alpha}}^{\hat{\beta}}, \quad \delta S_{\alpha \hat{\gamma}}^{\beta \hat{\delta}}=\hat{C}_{\hat{\gamma}}^{\hat{\delta} \kappa} \delta \Omega_{\kappa \alpha}{ }^{\beta}+C_{\alpha}^{\beta \hat{\kappa}} \delta \hat{\Omega}_{\hat{\kappa} \hat{\gamma}} \hat{\delta}
\end{gathered}
$$

where $h^{c \delta}$ and $\hat{h}^{c \hat{\delta}}$ are independent local gauge parameters and the transformations of $\Omega_{\alpha \beta} \gamma$ and $\hat{\Omega}_{\hat{\alpha} \hat{\beta}} \hat{\gamma}$ have been chosen such that $\lambda^{\alpha} \delta d_{\alpha}=\hat{\lambda}^{\hat{\alpha}} \delta d_{\hat{\alpha}}=0$. Note that $d_{\alpha}$ and $\hat{d}_{\hat{\alpha}}$ can be treated as independent variables in (56) since $p_{\alpha}$ and $\hat{p}_{\hat{\alpha}}$ do not appear explicitly.

The first line of (56) is the standard Type II GS action, but the other lines are needed for BRST invariance. As will now be shown to lowest order in $\alpha^{\prime}$, nilpotence and holomorphicity of $\lambda^{\alpha} d_{\alpha}$ and nilpotence and antiholomorphicity of $\hat{\lambda}^{\hat{\alpha}} \hat{d}_{\hat{\alpha}}$ imply the equations of motion for the background superfields in (56).

\subsection{Type II nilpotency constraints}

To analyze the conditions implied by nilpotency of $Q=\oint \lambda^{\alpha} d_{\alpha}$ and $\hat{Q}=\oint \hat{\lambda}^{\hat{\alpha}} \hat{d}_{\hat{\alpha}}$, it is convenient to use the canonical momenta $P_{M}=\partial L / \partial\left(\partial_{0} Z^{M}\right)$ to write

$$
\begin{aligned}
& d_{\alpha}=E_{\alpha}^{M}\left[P_{M}+\frac{1}{2} B_{M N}\left(\partial Z^{N}-\bar{\partial} Z^{N}\right)-\Omega_{M \beta}{ }^{\gamma} \lambda^{\beta} w_{\gamma}-\hat{\Omega}_{M \hat{\beta}} \hat{\gamma}^{\hat{\lambda}} \hat{\lambda}^{\hat{\beta}} \hat{w}_{\hat{\gamma}}\right], \\
& \hat{d}_{\hat{\alpha}}=E_{\hat{\alpha}}^{M}\left[P_{M}+\frac{1}{2} B_{M N}\left(\partial Z^{N}-\bar{\partial} Z^{N}\right)-\Omega_{M \beta}{ }^{\gamma} \lambda^{\beta} w_{\gamma}-\hat{\Omega}_{M \hat{\beta}} \hat{\gamma}^{\hat{\lambda}} \hat{\lambda}^{\hat{\beta}} \hat{w}_{\hat{\gamma}}\right] .
\end{aligned}
$$

Using the canonical commutation relations

$$
\left[P_{M}, Z^{N}\right\}=\delta_{M}^{N}, \quad\left[w_{\alpha}, \lambda^{\beta}\right]=\delta_{\alpha}^{\beta}, \quad\left[\hat{w}_{\hat{\alpha}}, \hat{\lambda}^{\hat{\beta}}\right]=\delta_{\hat{\alpha}}^{\hat{\beta}},
$$

one finds that

$$
\begin{aligned}
& \{Q, Q\}=\oint \lambda^{\alpha} \lambda^{\beta}\left[T_{\alpha \beta}{ }^{C} D_{C}+\frac{1}{2}\left(\partial Z^{N}-\bar{\partial} Z^{N}\right) H_{\alpha \beta N}-R_{\alpha \beta \gamma}{ }^{\delta} \lambda^{\gamma} w_{\delta}-\hat{R}_{\alpha \beta \hat{\gamma}} \hat{\hat{\delta}} \hat{\lambda}^{\hat{\gamma}} \hat{w}_{\hat{\delta}}\right] \\
& \{\hat{Q}, \hat{Q}\}=\oint \hat{\lambda}^{\hat{\alpha}} \hat{\lambda}^{\hat{\beta}}\left[T_{\hat{\alpha} \hat{\beta}}{ }^{C} D_{C}+\frac{1}{2}\left(\partial Z^{N}-\bar{\partial} Z^{N}\right) H_{\hat{\alpha} \hat{\beta} N}-R_{\hat{\alpha} \hat{\beta} \gamma}{ }^{\delta} \lambda^{\gamma} w_{\delta}-\hat{R}_{\hat{\alpha} \hat{\beta} \hat{\gamma}} \hat{\hat{\gamma}} \hat{\lambda}^{\hat{\gamma}} \hat{w}_{\hat{\delta}}\right] \\
& \{Q, \hat{Q}\}=\oint \lambda^{\alpha} \hat{\lambda}^{\hat{\beta}}\left[T_{\alpha \hat{\beta}}{ }^{C} D_{C}+\frac{1}{2}\left(\partial Z^{N}-\bar{\partial} Z^{N}\right) H_{\alpha \hat{\beta} N}-R_{\alpha \hat{\beta} \gamma}{ }^{\delta} \lambda^{\gamma} w_{\delta}-\hat{R}_{\alpha \hat{\beta} \hat{\gamma}} \hat{\hat{\delta}} \hat{\gamma} \hat{w}_{\hat{\delta}}\right]
\end{aligned}
$$


where $D_{C}=E_{C}^{M}\left(P_{M}-\Omega_{M \alpha}{ }^{\beta} \lambda^{\alpha} w_{\beta}-\hat{\Omega}_{M \hat{\alpha}}{ }^{\hat{\beta}} \hat{\lambda}^{\hat{\alpha}} \hat{w}_{\hat{\beta}}\right), T_{A B}{ }^{\alpha}$ and $R_{A B \beta}{ }^{\gamma}$ are defined using the $\Omega_{M \beta}{ }^{\gamma}$ spin connection, and $T_{A B}{ }^{\hat{\alpha}}$ and $\hat{R}_{A B \hat{\beta}}{ }^{\hat{\gamma}}$ are defined using the $\hat{\Omega}_{M \hat{\beta}} \hat{\gamma}^{\gamma}$ spin connection.

So nilpotency of $Q$ and $\hat{Q}$ implies that

$$
\begin{gathered}
\lambda^{\alpha} \lambda^{\beta} T_{\alpha \beta}{ }^{C}=\lambda^{\alpha} \lambda^{\beta} H_{\alpha \beta B}=\lambda^{\alpha} \lambda^{\beta} \hat{R}_{\alpha \beta \hat{\gamma}}{ }^{\hat{\delta}}=\lambda^{\alpha} \lambda^{\beta} \lambda^{\gamma} R_{\alpha \beta \gamma}{ }^{\delta}=0, \\
\hat{\lambda}^{\hat{\alpha}} \hat{\lambda}^{\hat{\beta}} T_{\hat{\alpha} \hat{\beta}}{ }^{C}=\hat{\lambda}^{\hat{\alpha}} \hat{\lambda}^{\hat{\beta}} H_{\hat{\alpha} \hat{\beta} B}=\hat{\lambda}^{\hat{\alpha}} \hat{\lambda}^{\hat{\beta}} \hat{R}_{\hat{\alpha} \hat{\beta} \gamma}{ }^{\delta}=\hat{\lambda}^{\alpha} \hat{\lambda}^{\beta} \hat{\lambda}^{\hat{\gamma}} R_{\hat{\alpha} \hat{\beta} \hat{\gamma}}{ }^{\hat{\delta}}=0, \\
T_{\alpha \hat{\beta}}{ }^{C}=H_{\alpha \hat{\beta} B}=\lambda^{\alpha} \lambda^{\beta} R_{\alpha \hat{\gamma} \beta}{ }^{\delta}=\hat{\lambda}^{\hat{\alpha}} \hat{\lambda}^{\hat{\beta}} \hat{R}_{\gamma \hat{\alpha} \hat{\beta}}{ }^{\hat{\delta}}=0,
\end{gathered}
$$

for any pure spinors $\lambda^{\alpha}$ and $\hat{\lambda}^{\hat{\alpha}}$ satisfying (42). As in the heterotic case, the nilpotency constraints on $R_{A B C}{ }^{D}$ are implied through Bianchi identities by the nilpotency constraints on $T_{A B}{ }^{C}$.

As will be discussed in section 4, the constraints of (63) can be interpreted as Type II pure spinor integrability conditions and imply all the essential Type II supergravity constraints. The remaining conventional Type II supergravity constraints will be implied by the holomorphicity and antiholomorphicity of $\lambda^{\alpha} d_{\alpha}$ and $\hat{\lambda}^{\hat{\alpha}} \hat{d}_{\hat{\alpha}}$.

\subsection{Type II holomorphicity constraints}

To derive the constraints coming from holomorphicity of $\lambda^{\alpha} d_{\alpha}$ and antiholomorphicity of $\hat{\lambda}^{\alpha} \hat{d}_{\alpha}$, first vary $\lambda^{\alpha}, w_{\alpha}, \hat{\lambda}^{\hat{\alpha}}$ and $\hat{w}_{\hat{\alpha}}$ in (56) to obtain the equations

$$
\begin{gathered}
\bar{\partial} \lambda^{\alpha}=-\left(\Omega_{M \beta}^{\alpha} \bar{\partial} Z^{M}+C_{\beta}^{\alpha \hat{\gamma}} \hat{d}_{\hat{\gamma}}+S_{\beta \hat{\gamma}}^{\alpha \hat{\delta}} \hat{\lambda}^{\hat{\gamma}} \hat{w}_{\hat{\delta}}\right) \lambda^{\beta}, \\
\bar{\partial} w_{\alpha}=\left(\Omega_{M \alpha}^{\beta} \bar{\partial} Z^{M}+C_{\alpha}^{\beta \hat{\gamma}} \hat{d}_{\hat{\gamma}}+S_{\alpha \hat{\gamma}}^{\beta \hat{\delta}} \hat{\lambda} \hat{\gamma} \hat{w}_{\hat{\delta}}\right) w_{\beta}, \\
\partial \hat{\lambda}^{\hat{\alpha}}=-\left(\hat{\Omega}_{M \hat{\beta}}^{\hat{\alpha}} \partial Z^{M}+\hat{C}_{\hat{\beta}}^{\hat{\alpha} \gamma} d_{\gamma}+S_{\gamma \hat{\beta}}^{\delta \hat{\alpha}} \lambda^{\gamma} w_{\delta}\right) \hat{\lambda}^{\hat{\beta}}, \\
\partial \bar{w}_{\hat{\alpha}}=\left(\hat{\Omega}_{M \hat{\alpha}}^{\hat{\beta}} \partial Z^{M}+\hat{C}_{\hat{\alpha}}^{\hat{\beta} \gamma} d_{\gamma}+S_{\gamma \hat{\alpha}}^{\delta \hat{\beta}} \lambda w_{\delta}\right) \hat{w}_{\hat{\beta}} .
\end{gathered}
$$

And by computing $E_{\alpha}^{P}\left(\delta S / \delta Z^{P}\right)$, one obtains the equation of motion

$$
\begin{gathered}
\bar{\partial} d_{\alpha}=E_{\alpha}^{P}\left[\left(\partial_{[P} E_{M]}^{a} E_{N}^{b} \eta_{a b}+\partial_{[P} E_{N]}^{a} E_{M}^{b} \eta_{a b}+\frac{1}{2} H_{P M N}\right) \partial Z^{M} \bar{\partial} Z^{N}\right. \\
+2\left(\partial_{[P} E_{N]}^{\beta} d_{\beta}+\partial_{[P} \omega_{N] \alpha}^{\beta} \lambda^{\alpha} w_{\beta}\right) \bar{\partial} Z^{N}+2\left(\partial_{[P} E_{N]}^{\hat{\beta}} \hat{d}_{\hat{\beta}}+\partial_{[P} \hat{\Omega}_{N] \hat{\alpha}}{ }^{\hat{\beta}} \hat{\lambda}^{\hat{\alpha}} \hat{w}_{\hat{\beta}}\right) \partial Z^{N} \\
-\Omega_{P \alpha}{ }^{\beta} \bar{\partial}\left(\lambda^{\alpha} w_{\beta}\right)-\hat{\Omega}_{P \hat{\alpha}} \hat{\beta} \partial\left(\hat{\lambda}^{\hat{\alpha}} \hat{w}_{\hat{\beta}}\right) \\
\left.+\partial_{P} P^{\alpha \hat{\beta}} d_{\alpha} \hat{d}_{\hat{\beta}}+\partial_{P} C_{\alpha}^{\beta \hat{\gamma}}(Z) \lambda^{\alpha} w_{\beta} \hat{d}_{\hat{\gamma}}+\partial_{P} \hat{C}_{\hat{\alpha}}^{\hat{\beta} \gamma}(Z) \hat{\lambda}^{\hat{\alpha}} \hat{w}_{\hat{\beta}} d_{\gamma}+\partial_{P} S_{\alpha \hat{\gamma}}^{\beta \hat{\delta}}(Z) \lambda^{\alpha} w_{\beta} \hat{\lambda}^{\hat{\gamma}} \hat{w}_{\hat{\delta}}\right] .
\end{gathered}
$$

Putting these equations together, one finds

$$
\begin{gathered}
\bar{\partial}\left(\lambda^{\alpha} d_{\alpha}\right)=\lambda^{\alpha}\left[\frac{1}{2} T_{\alpha B c}\left(\Pi^{B} \bar{\Pi}^{c}+\Pi^{c} \bar{\Pi}^{B}\right)+\frac{1}{2} H_{\alpha B C} \Pi^{B} \bar{\Pi}^{C}\right. \\
+T_{\alpha B}^{\beta} d_{\beta} \bar{\Pi}^{B}+T_{\alpha B}{ }^{\hat{\beta}} \Pi^{B} \hat{d}_{\hat{\beta}}+\left(\nabla_{\alpha} P^{\rho \hat{\gamma}}+C_{\alpha}^{\rho \hat{\gamma}}\right) d_{\rho} \hat{d}_{\hat{\gamma}}+R_{\alpha B \gamma}{ }^{\delta} \lambda^{\gamma} w_{\delta} \bar{\Pi}^{B}+\hat{R}_{\alpha B \hat{\gamma}} \hat{\delta}^{B} \Pi^{B} \hat{\lambda}^{\hat{\gamma}} \hat{w}_{\hat{\delta}}
\end{gathered}
$$




$$
\left.+\nabla_{\alpha} C_{\beta}^{\delta \hat{\gamma}} \lambda^{\beta} w_{\delta} \hat{d}_{\hat{\gamma}}+\nabla_{\alpha} \hat{C}_{\hat{\beta}}^{\hat{\gamma} \rho} d_{\rho} \hat{\lambda}^{\hat{\beta}} \hat{w}_{\hat{\gamma}}+\nabla_{\alpha} S_{\kappa \hat{\beta}}^{\gamma \hat{\delta}} \lambda^{\kappa} w_{\gamma} \hat{\lambda}^{\hat{\beta}} \hat{w}_{\hat{\delta}}+\left(\nabla_{\alpha} \hat{C}_{\hat{\beta}}^{\hat{\gamma} \rho}+S_{\alpha \hat{\beta}}^{\rho \hat{\gamma}}\right) d_{\rho} \hat{\lambda}^{\hat{\gamma}} \hat{w}_{\hat{\beta}}\right]
$$

where $\Pi^{A}=E_{M}^{A} \partial Z^{M}, \bar{\Pi}^{A}=E_{M}^{A} \bar{\partial} Z^{M}, T_{A B c}=\eta_{c d} T_{A B}{ }^{d}$, and all superspace derivatives acting on unhatted spinor indices are covariantized using the $\Omega_{M \alpha}{ }^{\beta}$ connection while all superspace derivatives acting on hatted spinor indices are covariantized using the $\hat{\Omega}_{M \hat{\alpha}} \hat{\beta}^{\hat{\beta}}$ connection. Furthermore, the torsion $T_{A B}{ }^{\alpha}$ and curvature $R_{A B \gamma}{ }^{\delta}$ are defined as in (31) using the $\Omega_{M \alpha}{ }^{\beta}$ connection whereas the torsion $T_{A B}{ }^{\hat{\alpha}}$ and curvature $\hat{R}_{A B \hat{\gamma}} \hat{\delta}$ are defined using the $\hat{\Omega}_{M \hat{\alpha}} \hat{\beta}^{\hat{\beta}}$ connection. Note that $T_{A b c}$ appears only in the combination $T_{\alpha(b c)}$. This combination is independent of the spin connections since $\Omega_{M}^{(s)}$ and $\hat{\Omega}_{M}^{(s)}$ only act on spinor indices and since $\Omega_{M}^{a b}$ and $\hat{\Omega}_{M}^{a b}$ are antisymmetric in their vector indices.

Plugging into (66) the equations of motion which come from varying $d_{\alpha}$ and $\hat{d}_{\hat{\alpha}}$,

$$
\bar{\Pi}^{\alpha}=-P^{\alpha \hat{\beta}} \hat{d}_{\hat{\beta}}-\hat{C}_{\hat{\beta}}^{\hat{\gamma} \alpha} \hat{\lambda}^{\hat{\beta}} \hat{w}_{\hat{\gamma}}, \quad \Pi^{\hat{\alpha}}=P^{\beta \hat{\alpha}} d_{\beta}-C_{\beta}^{\gamma \hat{\alpha}} \lambda^{\beta} w_{\gamma},
$$

one finds that holomorphicity of $\lambda^{\alpha} d_{\alpha}$ implies that

$$
\begin{gathered}
T_{\alpha(b c)}=H_{\alpha c d}=H_{\alpha \hat{\beta} \gamma}=T_{\alpha \beta c}+H_{\alpha \beta c}=T_{\alpha \hat{\beta} c}-H_{\alpha \hat{\beta} c}=0 \\
T_{\alpha c}{ }^{\beta}+T_{\alpha \hat{\gamma} c} P^{\beta \hat{\gamma}}=T_{\alpha c}^{\hat{\beta}}-T_{\alpha \gamma c} P^{\gamma \hat{\beta}}=T_{\alpha \beta} \hat{\gamma}-\frac{1}{2} H_{\alpha \beta \gamma} P^{\gamma \hat{\gamma}}=T_{\alpha \hat{\gamma}}{ }^{\beta}=0, \\
C_{\alpha}^{\gamma \hat{\beta}}+\nabla_{\alpha} P^{\gamma \hat{\beta}}-T_{\alpha \rho}^{\gamma} P^{\rho \hat{\beta}}=\hat{R}_{c \alpha \hat{\beta}} \hat{\gamma}+T_{\alpha \rho c} \hat{C}_{\hat{\beta}}^{\hat{\gamma} \rho}=\hat{R}_{\alpha \delta \hat{\beta}} \hat{\gamma}-\frac{1}{2} H_{\alpha \delta \rho} C_{\hat{\beta}}^{\hat{\gamma} \rho}=0, \\
S_{\alpha \hat{\gamma}}^{\rho \hat{\delta}}+\hat{R}_{\alpha \hat{\beta} \hat{\gamma}}{ }^{\hat{\delta}} P^{\rho \hat{\beta}}+\nabla_{\alpha} \hat{C}_{\hat{\gamma}}^{\hat{\delta} \rho}-T_{\alpha \beta} \hat{C}_{\hat{\gamma}}^{\hat{\delta} \beta}=0, \\
\lambda^{\alpha} \lambda^{\beta}\left(R_{c \alpha \beta}^{\gamma}+T_{\alpha \hat{\delta} c} C_{\beta}^{\gamma \hat{\delta}}\right)=\lambda^{\alpha} \lambda^{\beta} R_{\hat{\delta} \alpha \beta}^{\gamma}=0, \\
\lambda^{\alpha} \lambda^{\beta}\left(\nabla_{\alpha} C_{\beta}^{\delta \hat{\gamma}}-R_{\alpha \kappa \beta} \delta P^{\kappa \hat{\gamma}}\right)=\lambda^{\alpha} \lambda^{\beta}\left(\nabla_{\alpha} S_{\beta \hat{\gamma}}^{\rho \hat{\delta}}-\hat{R}_{\alpha \hat{\kappa} \hat{\gamma}} C_{\beta}^{\rho \hat{\kappa}}-R_{\alpha \kappa \beta}{ }^{\rho} \hat{C}_{\hat{\gamma}}^{\hat{\delta} \kappa}\right)=0,
\end{gathered}
$$

where the last two lines of equations must be satisfied for any pure spinor $\lambda^{\alpha}$. Antiholomorphicity of $\hat{\lambda}^{\hat{\alpha}} \hat{d}_{\hat{\alpha}}$ implies the hatted version of the above equations. The only subtle point is that it implies $T_{\hat{\alpha} \hat{\beta} c}-H_{\hat{\alpha} \hat{\beta} c}=T_{\hat{\alpha} \beta c}+H_{\hat{\alpha} \beta c}=0$, which together with the above equations implies that

$$
T_{\alpha \beta c}+H_{\alpha \beta c}=T_{\hat{\alpha} \hat{\beta} c}-H_{\hat{\alpha} \hat{\beta} c}=T_{\alpha \hat{\beta} c}=H_{\alpha \hat{\beta} c}=0 .
$$

The constraints of (63) and (68) will now be shown to imply the correct Type II supergravity equations of motion.

\subsection{Type II supergravity constraints}

The analysis of the Type II constraints of (63) and (68) will closely resemble the analysis of the heterotic constraints in subsection (2.5). At scaling dimension $-\frac{1}{2}$, the constraints of (63) imply that

$$
H_{\alpha \beta \gamma}=H_{\alpha \beta \hat{\gamma}}=H_{\alpha \hat{\beta} \hat{\gamma}}=H_{\hat{\alpha} \hat{\beta} \hat{\gamma}}=0
$$

since there is no non-zero symmetric $H_{\alpha \beta \gamma}$ and $H_{\hat{\alpha} \hat{\beta} \hat{\gamma}}$ satisfying $\lambda^{\alpha} \lambda^{\beta} H_{\alpha \beta \gamma}=0$ and $\hat{\lambda}^{\hat{\alpha}} \hat{\lambda}^{\hat{\beta}} H_{\hat{\alpha} \hat{\beta} \hat{\gamma}}=$ 0 . 
At dimension 0 , the constraints $\lambda^{\alpha} \lambda^{\beta} T_{\alpha \beta}^{c}=\hat{\lambda}^{\hat{\alpha}} \hat{\lambda}^{\hat{\beta}} T_{\hat{\alpha} \hat{\beta}}^{c}=0$ imply that $T_{\alpha \beta}^{c}=i\left(\gamma^{d}\right)_{\alpha \beta} f_{d}^{c}$ and $T_{\hat{\alpha} \hat{\beta}}^{c}=i\left(\gamma^{d}\right)_{\hat{\alpha} \hat{\beta}} \hat{f}_{d}^{c}$ for some $f_{d}^{c}$ and $\hat{f}_{c}^{d}$. Using the dimension zero $\mathrm{H}$ Bianchi identities and the local Lorentz and scale transformations of (60) for the unhatted and hatted spinor indices independently, both $f_{d}^{c}$ and $\hat{f}_{d}^{c}$ can be gauge-fixed to $\delta_{d}^{c}$. After this gauge-fixing, the only remaining gauge invariance is a single local Lorentz invariance which acts on all spinor and vector indices in the standard fashion. Combining with the other dimension 0 constraints of (63) and (68), one has

$$
T_{\alpha \beta}^{c}=-\eta^{c d} H_{\alpha \beta d}=i\left(\gamma^{c}\right)_{\alpha \beta}, \quad T_{\hat{\alpha} \hat{\beta}}^{c}=\eta^{c d} H_{\hat{\alpha} \hat{\beta} d}=i\left(\gamma^{c}\right)_{\hat{\alpha} \hat{\beta}}, \quad T_{\alpha \hat{\beta}}^{c}=H_{\alpha \hat{\beta} c}=0 .
$$

At dimension $\frac{1}{2}$, the constraints $\lambda^{\alpha} \lambda^{\beta} T_{\alpha \beta} \gamma=0$ and $\hat{\lambda}^{\hat{\alpha}} \hat{\lambda}^{\hat{\beta}} T_{\hat{\alpha} \hat{\beta}^{\hat{\gamma}}}=0$ imply that $T_{\alpha \beta}{ }^{\gamma}=$ $f_{c}^{\gamma}\left(\gamma^{c}\right)_{\alpha \beta}$ and $T_{\hat{\alpha} \hat{\beta}} \hat{\gamma}=\hat{f}_{c}^{\hat{\gamma}}\left(\gamma^{c}\right)_{\hat{\alpha} \hat{\beta}}$ for some $f_{c}^{\gamma}$ and $\hat{f}_{c}^{\hat{\gamma}}$. Using the shift symmetries of $(27)$, both $f_{c}^{\gamma}$ and $\hat{f}_{c}^{\hat{\gamma}}$ can be gauge-fixed to zero so that $T_{\alpha \beta}{ }^{\gamma}=T_{\hat{\alpha} \hat{\beta}} \hat{\gamma}=0$. The other dimension $\frac{1}{2}$ constraints,

$$
\begin{aligned}
& H_{\alpha c d}=T_{\alpha(c d)}=T_{\alpha \beta} \hat{\gamma}=T_{\alpha \hat{\beta}} \gamma=0, \\
& H_{\hat{\alpha} c d}=T_{\hat{\alpha}(c d)}=T_{\hat{\alpha} \hat{\beta}} \gamma=T_{\hat{\alpha} \beta} \hat{\gamma}=0,
\end{aligned}
$$

imply through the Bianchi identities $(\nabla T+T T)_{\alpha \beta \gamma}^{c}=0$ and $(\nabla T+T T)_{\hat{\alpha} \hat{\beta} \hat{\gamma}}^{c}=0$ that

$$
T_{\gamma b}^{c}=2 \eta^{c d}\left(\gamma_{b d}\right)_{\alpha}{ }^{\beta} \Omega_{\beta}^{(s)}, \quad \hat{T}_{\hat{\gamma} b}^{c}=2 \eta^{c d}\left(\gamma_{b d}\right)_{\hat{\alpha}}{ }^{\hat{\beta}} \hat{\Omega}_{\hat{\beta}}^{(s)}
$$

where $T_{\gamma b}^{c}$ is defined using the $\Omega_{M}^{b c}$ spin connection and $\hat{T}_{\hat{\gamma} b}{ }^{c}$ is defined using the $\hat{\Omega}_{M}^{b c}$ spin connection. Furthermore, the Bianchi identities $(\nabla T+T T)_{\alpha \hat{\beta} \hat{\gamma}}^{c}=0$ and $(\nabla T+T T)_{\hat{\alpha} \beta \gamma}^{c}=0$ imply that

$$
\hat{T}_{\alpha b}{ }^{c}=T_{\hat{\alpha} b}{ }^{c}=\Omega_{\hat{\alpha}}^{(s)}=\hat{\Omega}_{\alpha}^{(s)}=0
$$

where $\hat{T}_{\alpha b}^{c}$ is defined using the $\hat{\Omega}_{M}^{b c}$ spin connection and $T_{\hat{\alpha} b}^{c}$ is defined using the $\Omega_{M}^{b c}$ spin connection.

At dimension one, the constraint $T_{c \alpha}{ }^{\beta}=T_{c \hat{\alpha}}{ }^{\hat{\beta}}=0$ decomposes into

$$
\begin{aligned}
& T_{c \alpha}{ }^{\beta}=T_{c}^{\text {defg }}\left(\gamma_{\text {defg }}\right)_{\alpha}{ }^{\beta}+T_{c}^{d e}\left(\gamma_{d e}\right)_{\alpha}{ }^{\beta}+T_{c} \delta_{\alpha}^{\beta}=0, \\
& T_{c \hat{\alpha}}{ }^{\hat{\beta}}=\hat{T}_{c}^{\text {defg }}\left(\gamma_{d e f g}\right)_{\hat{\alpha}}^{\hat{\beta}}+\hat{T}_{c}^{d e}\left(\gamma_{d e}\right)_{\hat{\alpha}}^{\hat{\beta}}+\hat{T}_{c} \delta_{\hat{\alpha}}^{\hat{\beta}}=0 .
\end{aligned}
$$

The constraints $T_{c}=\hat{T}_{c}=0$ and $T_{c}^{d e}=\hat{T}_{c}^{d e}=0$ determine the vector components of the spin connections $\Omega_{c}^{(s)}, \hat{\Omega}_{c}^{(s)}, \Omega_{c}{ }^{d e}$ and $\hat{\Omega}_{c}^{d e}$, whereas the constraint $T_{c}^{\text {defg }}=\hat{T}_{c}^{\text {defg }}=0$ is implied by the Bianchi identities $(D H+T H)_{b c \alpha \gamma}\left(\gamma^{b d e f g}\right)^{\alpha \gamma}=0$ and $(D H+T H)_{b c \hat{\alpha} \hat{\gamma}}\left(\gamma^{b d e f g}\right)^{\hat{\alpha} \hat{\gamma}}=0$. The constraints $T_{\alpha c}{ }^{\hat{\beta}}=\left(\gamma_{c}\right)_{\alpha \gamma} P^{\gamma \hat{\beta}}$ and $T_{\hat{\alpha} c}{ }^{\beta}=\left(\gamma_{c}\right)_{\hat{\alpha} \hat{\gamma}} \hat{P}^{\beta \hat{\gamma}}$ for some $P^{\gamma \hat{\beta}}$ and $\hat{P}^{\beta \hat{\gamma}}$ are implied by the Bianchi identities $(\nabla T+T T)_{\alpha \beta \gamma}^{\hat{\delta}}=(\nabla T+T T)_{\hat{\alpha} \hat{\beta} \hat{\gamma}}^{\delta}=0$. And $P^{\gamma \hat{\beta}}=\hat{P}^{\gamma \hat{\beta}}$ is implied by the Bianchi identity $(\nabla T+T T)_{\alpha \hat{\beta} c}^{c}=0$. Similarly, all other constraints in (63) and (68) are either implied by Bianchi identities or define $C_{\alpha}^{\beta \hat{\gamma}}, \hat{C}_{\hat{\alpha}}^{\hat{\beta} \gamma}$ and $S_{\beta \hat{\delta}}^{\alpha \hat{\gamma}}$ in terms of the supervielbein.

The above constraints imply that all background superfields appearing in the action of (56) can be expressed in terms of the spinor supervielbein $E_{\alpha}^{M}$ and $E_{\hat{\alpha}}^{M}$. Furthermore, the constraints

$$
T_{\alpha \beta}^{c}=i\left(\gamma^{c}\right)_{\alpha \beta}, \quad T_{\hat{\alpha} \hat{\beta}}^{c}=i\left(\gamma^{c}\right)_{\hat{\alpha} \hat{\beta}}, \quad T_{\alpha \hat{\beta}}^{c}=0
$$


imply the on-shell equations of motion for $E_{\alpha}^{M}$ and $E_{\hat{\alpha}}^{M}$. So the constraints of (63) and (68) imply the Type II supergravity equations of motion. In the following section, the above pure spinor description of Type IIB supergravity will be related to the Howe-West (HW) description of [30]. It should similarly be possible to relate the pure spinor description of Type IIA supergravity to the IIA superspace description of [33].

\section{Relation with the $S L(2, R)$ Covariant Description of IIB Su- pergravity}

In this section we shall demonstrate that the constraints on the torsion for IIB derived in the preceeding section are indeed equivalent to the HW equations of motion of IIB supergravity described in [30]. We shall do this by first showing that the latter are generated by the standard dimension zero torsion constraint and then exhibiting the explicit transformation from the standard IIB superspace torsions to those derived above. In order to carry through the first step we use the method of Weyl superspace and then we reduce the structure group to the Lorentz group. In order to establish the result fully we also have to examine the scalars in the theory.

The complete IIB supergravity theory was derived from a superspace perspective in [30]. However, although complete results were given there for all of the superspace field strength tensors, no attempt was made to identify a minimal generating set of constraints. Moreover, the HW formalism is manifestly locally $U(1)$ and globally $S L(2, \mathbb{R})$ invariant and this is not convenient for the applications we have in mind here. We shall work initially in an $S O(2)$ formalism (rather than $U(1)$ ) since this will be easier to adapt to our purposes.

For IIB superspace we use the same HW conventions as in [30], although we use $\gamma$ to denote the $16 \times 16$ spin matrices instead of $\sigma$. To convert $S O(2)$ spinor indices $i, j, \ldots$ to $U(1)$ indices, we write

$$
v^{i} \rightarrow v^{ \pm}=\frac{1}{\sqrt{2}}\left(v^{1} \pm v^{2}\right)
$$

and

$$
v_{i} \rightarrow v_{ \pm}=\frac{1}{\sqrt{2}}\left(v^{1} \mp v^{2}\right) .
$$

So the metric and $\epsilon$-tensor are

$$
\delta_{+-}=1, \quad \epsilon^{+-}=-i, \quad \epsilon_{+-}=i, \quad \epsilon_{+}^{+}=i .
$$

The summation is therefore $u^{i} v_{i}=u^{+} v_{+}+u^{-} v_{-} .{ }^{4}$ To convert $S O(2)$ vector indices $r, s, \ldots$ to $U(1)$ indices, we have

$$
v_{r}=\left(\tau_{r}\right)^{i j} v_{i j} \leftrightarrow v_{i j}=\left(\tau^{r}\right)_{i j} v_{r}
$$

where

$$
\tau^{r}=\frac{1}{\sqrt{2}}\left(\sigma_{3}, \sigma_{1}\right)
$$

\footnotetext{
${ }^{4}$ This causes a slight problem in the superspace summation convention which should be taken to be $u^{\alpha i} v_{\alpha i}=$ $u^{\alpha+} v_{\alpha+}+u^{\alpha-} v_{\alpha-}$, whereas in [30] one finds $u^{\alpha} v_{\alpha}-u^{\bar{\alpha}} v_{\bar{\alpha}}$. So, in converting from HW conventions to $S O(2)$, one has to remember to insert an extra minus sign for downstairs $\alpha$ - indices. This means, for example, that we must take $T_{\alpha i \beta j}{ }^{c}=i \delta_{i j}\left(\gamma^{c}\right)_{\alpha \beta}$ since then one finds $T_{\alpha+\beta-}{ }^{c}=i\left(\gamma^{c}\right)_{\alpha \beta} \Rightarrow T_{\alpha \bar{\beta}^{c}}{ }^{c}=-i\left(\gamma^{c}\right)_{\alpha \beta}$ in agreement with [30].
} 
We can then put

$$
v^{ \pm \pm}=\frac{1}{\sqrt{2}}\left(v^{1} \pm v^{2}\right)
$$

for vector indices and this is consistent since $\left(\tau^{++}\right)_{++}=1$.

In subsection (4.1), we shall first show that the equations of motion of IIB supergravity follow (up to topological niceties) from the usual dimension zero constraint

$$
T_{\alpha i \beta j}{ }^{c}=i \delta_{i j}\left(\gamma^{c}\right)_{\alpha \beta} .
$$

We shall do this by working in Weyl superspace, i.e. we shall include a scale factor in the structure group. Following through the consequences of this we find that the scale curvature vanishes so that the scale connection is pure gauge. If we then take it to vanish we recover the equations of [30]. This procedure is very similar to the approach used in [31] to prove that the equations of motion of $D=11$ supergravity follow from the standard dimension zero constraint.

Since the standard dimension zero constraint of (83) is required by the nilpotency of $Q$, it then follows that the equations of motion of IIB supergravity are indeed implied in the pure spinor formalism. However, as we have seen, there are many other equations at dimensions greater than zero that are required to hold either by the nilpotency or by the holomorphicity of $Q$. In subsection (4.2), we shall check these explicitly at dimension one-half by comparing our results with those of section 3 .

In the HW superspace description of Type IIB supergravity, $S L(2, \mathbb{R})$ global symmetry is manifest since the two scalars are described by an $S O(2) \backslash S L(2, \mathbb{R})$ coset. However, this $S L(2, \mathbb{R})$ symmetry is not manifest in the pure spinor description since the dilaton and axion do not appear in an $S L(2, \mathbb{R})$ covariant manner. In subsection (4.3), we will relate these two descriptions of the Type IIB scalars and will show that the target-space metric appearing in the pure spinor version of the Type IIB sigma model is in string gauge.

\subsection{Weyl superspace}

To get the superspace constraints under control it is useful to include a scale factor in the connection. The structure group is then $\operatorname{Spin}(1,9) \times \operatorname{Spin}(2) \times \mathbb{R}^{+}$. The full connection (denoted by a tilde) is

$$
\begin{aligned}
\tilde{\Omega}_{a}{ }^{b} & =\Omega_{a}{ }^{b}+2 \delta_{a}{ }^{b} \Pi \\
\tilde{\Omega}_{\alpha i}{ }^{\beta j} & =\delta_{i}{ }^{j} \Omega_{\alpha}{ }^{\beta}+\delta_{\alpha}{ }^{\beta}\left(\delta_{i}{ }^{j} \Pi+\epsilon_{i}{ }^{j} \Sigma\right)
\end{aligned}
$$

where $\Omega, \Sigma, \Pi$ are respectively the connections for the Lorentz, $U(1)(=\operatorname{Spin}(2))$ and scale factors. We shall use the notation $\Omega^{\prime}$ to denote the $\operatorname{Spin}(1,9) \times U(1)$ connection, so $\Omega^{\prime} \sim \Omega+\Sigma$. Similarly, for the curvatures we have $\tilde{R} \sim R^{\prime}+M \sim R+M+N$ where $M$ and $N$ are respectively the $U(1)$ and scale curvatures.

At dimension one-half we find, using the Bianchi identity

$$
\tilde{D}_{(\alpha i} \tilde{T}_{\beta j \gamma k)}^{d}+\tilde{T}_{(\alpha i \beta j}^{E} \tilde{T}_{|E| \gamma k)}^{d}=0
$$


and the freedom to choose the dimension one-half components of the connection and the even basis vectors $E_{a}$, that the dimension one-half component of the torsion tensor is

$$
\tilde{T}_{\alpha i \beta j}{ }^{\gamma k}=-i\left(\gamma^{a} \gamma_{a}-2 \delta \delta\right)_{(\alpha \beta)}{ }^{\gamma \delta} \Lambda_{\delta i j}{ }^{k}
$$

where $\Lambda_{\alpha i j k}$ is totally symmetric and traceless on its $\operatorname{Spin}(2)$ indices, while

$$
\tilde{T}_{\alpha b}^{c}=0 .
$$

This is exactly the same as in [30], and we identify the HW spinor field $\Lambda$ by

$$
\begin{aligned}
& \Lambda=\sqrt{2}\left(\Lambda_{222}+i \Lambda_{111}\right)=i \Lambda_{---} \\
& \bar{\Lambda}=\sqrt{2}\left(\Lambda_{222}-i \Lambda_{111}\right)=-i \Lambda_{+++}
\end{aligned}
$$

At dimension one one has to solve two Bianchi identities

$$
\begin{aligned}
\tilde{R}_{\alpha i \beta j, c}{ }^{d} & =\tilde{T}_{\alpha i \beta j}{ }^{E} \tilde{T}_{E c}{ }^{d}+\tilde{T}_{c \alpha i}{ }^{\epsilon m} \tilde{T}_{\epsilon m \beta j}{ }^{d}+\tilde{T}_{c \beta j}{ }^{\epsilon m} \tilde{T}_{\epsilon m \alpha i}{ }^{d} \\
\tilde{R}_{(\alpha i \beta j, \gamma k)}{ }^{\delta l} & =\tilde{D}_{(\alpha i} \tilde{T}_{\beta j \gamma k)}{ }^{\delta l}+\tilde{T}_{(\alpha i \beta j}{ }^{e} \tilde{T}_{e \gamma k)}{ }^{\delta l}+\tilde{T}_{(\alpha i \beta j}{ }^{\epsilon m} \tilde{T}_{|\epsilon m| \gamma k)}{ }^{\delta l} .
\end{aligned}
$$

After a long and tedious calculation one can show that the only non-zero dimension one components of the curvature and torsion tensors are those which correspond to the dimension one components of the IIB supergravity multiplet, that is $F_{a b c}, P_{a}, G_{a b c d e}$ together with fermion bilinear terms. The tensors $F, P$ and $G$ are asociated with the antisymmetric tensor gauge fields of the theory and the scalar fields ( $P_{a}$ is essentially the derivative of the scalar fields). One also determines the spinorial derivative of $\Lambda$ and the dimension one component of the $U(1)$ curvature $M$ in terms of these physical fields. Moreover, one finds that the dimension one component of the scale curvature $N$ vanishes, $N_{\alpha i \beta j}=0$. From this, one immediately concludes with the aid of the scale curvature Bianchi identity, $d N=0$, that the whole of $N$ vanishes and so the scale connection is pure gauge as anticipated. At this stage we can set the scale connection equal to zero and recover the HW torsions and curvatures of [30]. From these results one can then construct super extensions of $F, G, P$ which satisfy corresponding Bianchi identities. In particular, one can deduce the existence of the two scalar fields described by an $S L(2, \mathbb{R}) \backslash U(1)$ coset space.

\subsection{Lorentz superspace}

To recover the form of the torsion and curvature tensors derived from the pure spinor formalism, we need firstly to restrict the structure group to be the ten-dimensional spin group. This means that the components of $\Pi$ and $\Sigma$ will appear in the redefined torsion. Moreover, we shall choose a different scale gauge from $\Pi=0$ which means that $\Pi=-d S$ for some scalar field $S$ and also that there is change of basis with respect to the HW basis, i.e. $E^{a}=e^{2 s} E_{H W}^{a}$, etc. Explicitly, we have

$$
\tilde{T}_{A B}^{C}=T_{A B}^{C}+2 \Pi_{[A} I_{B]}^{C}+2 \Sigma_{[A} J_{B]}^{C}
$$


where

$$
\begin{aligned}
I_{A}{ }^{B} & =\left\{\begin{array}{l}
I_{a}{ }^{b}=2 \delta_{a}{ }^{b} \\
I_{\alpha i}{ }^{\beta j}=\delta_{\alpha}{ }^{\beta} \delta_{i}{ }^{j}
\end{array}\right. \\
J_{A}{ }^{B} & =\left\{\begin{array}{l}
J_{a}{ }^{b}=0 \\
J_{\alpha i}{ }^{\beta j}=\delta_{\alpha}{ }^{\beta} \epsilon_{i}{ }^{j}
\end{array}\right.
\end{aligned}
$$

and where the mixed spinor-vector components of $I$ and $J$ are zero. In particular, at dimension one-half, we have

$$
\begin{aligned}
\tilde{T}_{\alpha i \beta j}{ }^{\gamma k} & =T_{\alpha i \beta j}{ }^{\gamma k}+\delta_{\alpha}{ }^{\gamma}\left(\delta_{i}{ }^{k} \Pi_{\beta j}+\epsilon_{i}{ }^{k} \Sigma_{\beta j}\right)+\delta_{\beta}{ }^{\gamma}\left(\delta_{j}{ }^{k} \Pi_{\alpha i}+\epsilon_{j}{ }^{k} \Sigma_{\alpha i}\right) \\
\tilde{T}_{\alpha i b}{ }^{c} & =T_{\alpha i b}{ }^{c}+2 \delta_{b}{ }^{c} \Pi_{\alpha i} .
\end{aligned}
$$

We shall also have to shift the Lorentz connection as

$$
\Omega_{\alpha b c} \rightarrow \Omega_{\alpha b c}^{(1,2)}=\Omega_{\alpha b c}+\left(\gamma_{b c}\right)_{\alpha}^{\beta} Y_{\beta}^{(1,2)} .
$$

The notation here is that the connection labelled $i=1,2$ will act on spinor indices with the same internal index label. Since the two connections will be different, this procedure manifestly breaks $S O(2)$. For the moment we shall suppose that the vector indices are acted upon by the original $\Omega$. Finally, in order to make a direct comparison to the earlier results we shall have to shift the vectorial basis $E_{a}$ by

$$
E_{a} \rightarrow E_{a}+i\left(\gamma^{a}\right)^{\alpha \beta} \chi_{\alpha i} E_{\beta i}
$$

If we choose

$$
\begin{aligned}
Y_{\alpha 1}^{(1)} & =i \Lambda_{\alpha 111}, \\
Y_{\alpha 2}^{(1)} & =-i \Lambda_{\alpha 222}, \\
Y_{\alpha 1}^{(2)} & =-i \Lambda_{\alpha 111}, \\
Y_{\alpha 2}^{(2)} & =i \Lambda_{\alpha 222}, \\
\chi_{\alpha 1} & =-i \Lambda_{\alpha 111}, \\
\chi_{\alpha 2} & =-i \Lambda_{\alpha 222},
\end{aligned}
$$

and if, in addition,

$$
\begin{aligned}
\Sigma_{\alpha 1} & =-i \Lambda_{\alpha 222}, \\
\Sigma_{\alpha 2} & =i \Lambda_{\alpha 111}, \\
\Pi_{\alpha 1} & =-\frac{i}{2} \Lambda_{\alpha 111}, \\
\Pi_{\alpha 2} & =-\frac{i}{2} \Lambda_{\alpha 222},
\end{aligned}
$$

then we find that all components of the redefined $T_{\alpha i \beta j}{ }^{\gamma k}$ vanish except for

$$
\begin{aligned}
& T_{\alpha 1 \beta 1}{ }^{\gamma 1}=-2 \delta_{(\alpha}{ }^{\gamma} \Omega_{\beta)}^{(s)}, \\
& T_{\alpha 2 \beta 2}{ }^{\gamma 2}=-2 \delta_{(\alpha} \hat{\Omega}_{\beta)}^{(s)}
\end{aligned}
$$


where

$$
\Omega_{\alpha}^{(s)}=i \Lambda_{\alpha 111} ; \quad \hat{\Omega}_{\alpha}^{(s)}=i \Lambda_{\alpha 222} .
$$

If we also define new vectorial torsions with respect to the new connections $\Omega_{\alpha b c}^{(1,2)}$, we find

$$
\begin{aligned}
T_{\alpha 1 b}^{(1) c} & =2\left(\gamma_{b}^{c} \Omega^{(s)}\right)_{\alpha}, \\
T_{\alpha 2 b}^{(1) c} & =0 \\
T_{\alpha 1 b}^{(2) c} & =0 \\
T_{\alpha 2 b}^{(2) c} & =2\left(\gamma_{b}^{c} \hat{\Omega}^{(s)}\right)_{\alpha} .
\end{aligned}
$$

We shall verify that equations (99) are indeed satisfied in the next section. For the moment, assuming that they are, we are now in a position to compare directly with the dimension one-half results coming from the pure spinor formalism. In order to do this, we remove the fermionic scale connection from the type II structure group. After identifying the indices $(\alpha 1, \alpha 2)=(\alpha, \hat{\alpha})$, we find that the only non-vanishing components of the redefined torsion with three spinorial indices are those of (100). The vectorial torsions, which do not need to be redefined, are those of (102). We have therefore succeeded in demonstrating that the torsions derived from the pure spinor formalism are indeed in agreement with those of [30] after suitable field redefinitions. To complete the picture we must verify that $\Pi=-d S$ and that the expressions given for $\Sigma_{\alpha i}$ in (99) are correct. To do this, we need to examine the scalar fields in the theory.

\subsection{Scalar fields}

The scalar fields take their values in the space $S O(2) \backslash S L(2, \mathbb{R})$. We describe then by a real two by two matrix $\mathcal{U}$ acted on by $\mathcal{U} \rightarrow h \mathcal{U} g^{-1}$, for $h \in S O(2), g \in S L(2, \mathbb{R})$. In index notation we write $\mathcal{U}_{r}{ }^{R}$. Note that $r$ is vector $S O(2)$ index while $R$ is an $S L(2, \mathbb{R})$ doublet index. The Maurer-Cartan form $\mathcal{M}$ is given by

$$
\mathcal{M}=d \mathcal{U U}^{-1}
$$

Since it is Lie-algebra valued it can be written as

$$
\mathcal{M}_{r}^{s}=P_{r}^{s}+2 \epsilon_{r}^{s} \Sigma
$$

where $\Sigma$ is the $U(1)$ connection of (85) and $P^{r s}$ is symmetric traceless, i.e. in complex notation we have $P^{++++}:=P$ where $P$ is the HW one-form defined in [30]. The Maurer-Cartan equation, $d \mathcal{M}+\mathcal{M}^{2}=0$, implies that

$$
\begin{aligned}
D P & =0 \\
M & =-\frac{i}{2} P \wedge \bar{P}
\end{aligned}
$$

where $M$ is the $U(1)$ curvature tensor.

There is a $\mathrm{HW} S L(2 \mathbb{R})$ doublet of three-form field strength tensors $\tilde{F}_{R}$, and we define $F:=\mathcal{U} \tilde{F}$. Assuming that $d \tilde{F}=0$ we find that

$$
D F_{r}=P_{r}^{s} F_{s}
$$


As before, we can identify $F_{--}=F^{++}$with the field $F$ of [30]. In a complex basis (107) reads

$$
D F=P \wedge \bar{F}
$$

as in [30].

The field $\mathcal{U}$ is not quite the same as the HW field $\mathcal{V}$. The two are related by

$$
\mathcal{U}=\epsilon \mathcal{V}^{-1} \epsilon
$$

The Maurer-Cartan form is then

$$
\begin{aligned}
d \mathcal{U U}^{-1} & =\epsilon \mathcal{V}^{-1} d \mathcal{V} \epsilon \\
& =\left(\begin{array}{rr}
0 & 1 \\
-1 & 0
\end{array}\right)\left(\begin{array}{rr}
2 i \Sigma & P \\
\bar{P} & -2 i \Sigma
\end{array}\right)\left(\begin{array}{rr}
0 & 1 \\
-1 & 0
\end{array}\right) \\
& =\left(\begin{array}{rr}
2 i \Sigma & \bar{P} \\
P & -2 i \Sigma
\end{array}\right) .
\end{aligned}
$$

In the second line of (110), we have used the formula for the Maurer-Cartan form in [30] (with $\Sigma$ instead of $Q$ ), and in the final line we have the correct expression in the new conventions in a complex basis.

In the physical gauge we can write the components of $\mathcal{U}$ in terms of $\tau:=\tau_{1}+i \tau_{2}:=C_{0}+i e^{-\Phi}$ where $\Phi$ is the dilaton and $C_{o}$ is the axion. In the real basis we have

$$
\mathcal{U}=\frac{1}{\sqrt{\tau_{2}}}\left(\begin{array}{ll}
1 & \tau_{1} \\
0 & \tau_{2}
\end{array}\right)
$$

and one can check that $\tau$ has the expected transformation under $S L(2, \mathbb{R})$, i.e.

$$
\tau \rightarrow \frac{a \tau+b}{c \tau+d}
$$

where $g \in S L(2, \mathbb{R})$ is

$$
g=\left(\begin{array}{rr}
a & -b \\
-c & d
\end{array}\right)
$$

In a complex basis (for both indices)

$$
\mathcal{U}=\frac{1}{2 \sqrt{\tau_{2}}}\left(\begin{array}{ll}
1+i \bar{\tau} & 1-i \bar{\tau} \\
1+i \tau & 1-i \tau
\end{array}\right)
$$

If one computes the Maurer-Cartan form in this gauge one finds

$$
\begin{aligned}
P & =\frac{1}{2}\left(d \Phi+i e^{\Phi} d C_{o}\right)=\frac{i d \tau}{2 \tau_{2}}, \\
\Sigma & =\frac{1}{4} e^{\Phi} d C_{o} .
\end{aligned}
$$

In the HW description, $P^{++++}$is related to $\Lambda_{\alpha}^{j k l}$ of (87) by

$$
P^{++++}=2 E^{\alpha+} \Lambda_{\alpha}^{+++}+E^{a} P_{a}^{++++}
$$


and $\tau$ is chiral, i.e. $\bar{D} \tau=0$. This implies

$$
\begin{aligned}
& D_{\alpha 1} C_{o}=-e^{-\Phi} D_{\alpha 2} \Phi \\
& D_{\alpha 2} C_{o}=e^{-\Phi} D_{\alpha 1} \Phi
\end{aligned}
$$

Using this we can express the components of $\Lambda$ as

$$
\begin{aligned}
\Lambda_{\alpha 111} & =-\frac{i}{4} D_{\alpha 1} \Phi \\
\Lambda_{\alpha 222} & =-\frac{i}{4} D_{\alpha 2} \Phi .
\end{aligned}
$$

We can also express the components of $\Sigma$ in terms of $D \Phi$ as

$$
\begin{aligned}
\Sigma_{\alpha 1} & =-\frac{1}{4} D_{\alpha 2} \Phi \\
\Sigma_{\alpha 2} & =\frac{1}{4} D_{\alpha 1} \Phi .
\end{aligned}
$$

Now earlier we found what the $U(1)$ and scale connections had to be chosen to be at dimension one-half in order to achieve $Q$-integrability. We required

$$
\begin{aligned}
\Sigma_{\alpha 1}^{\prime} & =-i \Lambda_{\alpha 222}^{\prime}, \\
\Sigma_{\alpha 2}^{\prime} & =i \Lambda_{\alpha 111}^{\prime} \\
\Pi_{\alpha 1}^{\prime} & =\frac{-i}{2} \Lambda_{\alpha 111}^{\prime} \\
\Pi_{\alpha 2}^{\prime} & =\frac{-i}{2} \Lambda_{\alpha 222}^{\prime}
\end{aligned}
$$

where $\Pi$ is the scale connection and where the prime indicates the basis which is related to the unprimed HW one by $E^{\prime \alpha i}=e^{S} E^{\alpha i}, E^{\prime a}=e^{2 S} E^{a}$. We also required $\Pi=-d S$, since it is pure gauge. So we can identify

$$
S=\frac{\Phi}{8}
$$

In addition, if we compare the expressions for the components of $\Lambda$ and $\Sigma$ in terms of $D \Phi$, we see that they agree, and so everything works as expected. If we define bosonic metrics by

$$
\begin{aligned}
G & =E^{b} \otimes E^{a} \eta_{a b}, \\
G^{\prime} & =E^{\prime b} \otimes E^{\prime a} \eta_{a b},
\end{aligned}
$$

then $G^{\prime}=e^{\frac{\Phi}{2}} G$. This means we can identify $G^{\prime}$ with the string metric and $G$ with the Einstein metric, so the conformal transformation we need to make is precisely the one which goes between the two frames.

\section{$5 \quad$ Higher Order $\alpha^{\prime}$ Corrections}

In this paper we have verified to lowest order in $\alpha^{\prime}$ that nilpotence and holomorphicity of the pure spinor BRST operator implies the superspace equations of motion for the background 
supergravity fields. The next question to investigate is how these superspace equations of motion are modified by higher order $\alpha^{\prime}$ corrections to the nilpotency and holomorphicity conditions. Since the sigma model is a free action in a flat background, one can compute these corrections using standard sigma model methods by separating the worldsheet variables into classical and quantum parts and expanding in normal coordinates around a flat background.

When the background fields satisfy their string-corrected equations of motion, one expects that the $\beta$-functions of the sigma model should vanish, i.e. that the sigma model remains conformally invariant at the quantum level. However, unlike the bosonic string sigma model, quantum conformal invariance is not expected to imply the complete set of equations of motion for the background fields. In addition, one needs to impose the conditions that, at the conformal fixed point, $\lambda^{\alpha} d_{\alpha}$ is holomorphic and nilpotent. It should be possible to impose these nilpotence and holomorphicity conditions perturbatively in $\alpha^{\prime}$ by computing contributions of the quantum worldsheet variables and the Fradkin-Tseytlin term to the equations of motion and OPE's of $\lambda^{\alpha}$ and $d_{\alpha}$.

The necessity of imposing BRST nilpotence and holomorphicity can be seen at the linearized level by analyzing the superstring vertex operators of (17), (18) and (53). When the superfields in these vertex operators are on-shell to linearized order, one can check that the vertex operators have no poles with the stress tensor $T$ and therefore preserve quantum conformal invariance. However, the condition of having no poles with $T$ is weaker than BRST invariance (i.e. $\left.\left[Q_{\text {flat }}, V\right]=0\right)$ and does not imply the complete set of linearized on-shell conditions. Note that $Q=Q_{\text {flat }}+V$ to linearized level, so $\left[Q_{\text {flat }}, V\right]=0$ implies that $Q$ is nilpotent to linearized order.

Besides the Chern-Simons modifications to the three-form field strength, the first superstring corrections to the supergravity equations of motion are expected to come at order $\left(\alpha^{\prime}\right)^{3}$, e.g. from the $R^{4}$ term. Since the supergravity equations of motion are implied by classical nilpotence and holomorphicity of the BRST operator, one expects to see these $\left(\alpha^{\prime}\right)^{3}$ corrections to the equations at three loops in the nilpotence and holomorphicity conditions. However, already at first order in $\alpha^{\prime}$, there are several non-trivial one-loop contributions to the nilpotence and holomorphicity conditions which must be cancelled by contributions from the Fradkin-Tseytlin term and from the Chern-Simons modification to the three-form field strength.

For example, for the heterotic superstring, the term $E_{\alpha}^{P}\left(-\Omega_{P \gamma}{ }^{\beta} \bar{\partial}\left(\lambda^{\gamma} w_{\beta}\right)-A_{P I} \partial \bar{J}^{I}\right)$ appearing in $\bar{\partial} d_{\alpha}$ in equation (36) gets one-loop corrections from the chiral anomalies

$$
\begin{gathered}
\partial \bar{J}^{I}=\frac{1}{2} \alpha^{\prime} \partial_{[M} A_{N]}^{I} \partial Z^{M} \bar{\partial} Z^{N} \\
\bar{\partial}\left(\lambda^{\gamma} w_{\beta}\right)=-\frac{1}{2} \alpha^{\prime} \partial_{[M} \Omega_{N] \beta}^{\gamma} \partial Z^{M} \bar{\partial} Z^{N}+\frac{1}{8} \alpha^{\prime} r \delta_{\beta}^{\gamma}
\end{gathered}
$$

where $r$ is the worldsheet curvature and the coefficient $\frac{1}{8} \alpha^{\prime}$ in (132) can be obtained by computing the coefficient of the triple pole of $\lambda^{\gamma} w_{\beta}$ with the pure spinor stress tensor $T_{\lambda}$ and dividing by 
four. ${ }^{5}$ So $\bar{\partial} d_{\alpha}$ gets a one-loop contribution

$$
-2 \alpha^{\prime} r E_{\alpha}^{P} \Omega_{P}^{(s)}+\frac{1}{2} \alpha^{\prime} E_{\alpha}^{P}\left(\Omega_{P \gamma}{ }^{\beta} \partial_{[M} \Omega_{N] \beta}^{\gamma}-A_{P}^{I} \partial_{[M} A_{N]}^{I}\right) \partial Z^{M} \bar{\partial} Z^{N} .
$$

After including other one-loop contributions coming from contractions of the quantum worldsheet variables, one expects that the second term in (133) is completed to $\frac{1}{2} \alpha^{\prime} E_{\alpha}^{P} w_{P M N}^{(C S)} \partial Z^{M} \bar{\partial} Z^{N}$ where

$$
w_{P M N}^{(C S)}=3 \operatorname{Tr}\left(\Omega_{[P} \partial_{M} \Omega_{N]}+\frac{2}{3} \Omega_{[P} \Omega_{M} \Omega_{N]}-A_{[P} \partial_{M} A_{N]}-\frac{2}{3} A_{[P} A_{M} A_{N]}\right)
$$

is the Chern-Simons three-form constructed from the gauge, scale and Lorentz connections.

The first term in (133) is cancelled by the contribution from the Fradkin-Tseytlin term

$$
\frac{1}{2 \pi \alpha^{\prime}} \int d^{2} z \frac{1}{2} \alpha^{\prime} r \Phi(Z)
$$

which contributes $\frac{1}{2} \alpha^{\prime} r D_{\alpha} \Phi$ to $\bar{\partial} d_{\alpha}$. So the $\alpha^{\prime} r$ contribution to $\bar{\partial} d_{\alpha}$ is cancelled if the heterotic dilaton superfield $\Phi$ is related to the scale connection $\Omega_{P}^{(s)}$ by

$$
D_{\alpha} \Phi=4 E_{\alpha}^{P} \Omega_{P}^{(s)},
$$

which can be checked to imply that the metric is in string gauge.

Since $\bar{\partial} d_{\alpha}$ of (36) also contains the term $\frac{1}{2} E_{\alpha}^{P} H_{P M N} \partial Z^{M} \bar{\partial} Z^{N}$, the second term in (133) can be cancelled by redefining

$$
H_{P M N} \rightarrow H_{P M N}-\alpha^{\prime} w_{P M N}^{(C S)} .
$$

As in the RNS sigma model [35], the need for redefining $H_{P M N}$ can also be seen by requiring gauge invariance of the sigma model action. Because of (131) and (132), the action of (24) is invariant under local gauge, scale and Lorentz transformations only if $B_{M N}$ is defined to transform as

$$
\delta B_{M N}=\alpha^{\prime}\left(\partial_{[M} A_{N]}^{I} \Lambda^{I}-\partial_{[M} \Omega_{N]}^{(s)} \Lambda^{(s)}-\partial_{[M} \Omega_{N]}^{a b} \Lambda_{a b}\right)
$$

where $\Lambda^{I}, \Lambda^{(s)}$ and $\Lambda^{a b}$ are the gauge parameters.

Similarly, for the Type II superstring, the anomalies

$$
\begin{gathered}
\bar{\partial}\left(\lambda^{\gamma} w_{\beta}\right)=-\frac{1}{2} \alpha^{\prime} \partial_{[M} \Omega_{N] \beta}{ }^{\gamma} \partial Z^{M} \bar{\partial} Z^{N}+\frac{1}{8} \alpha^{\prime} r \delta_{\gamma}^{\beta}, \\
\partial\left(\hat{\lambda}^{\hat{\gamma}} \hat{w}_{\hat{\beta}}\right)=\frac{1}{2} \alpha^{\prime} \partial_{[M} \hat{\Omega}_{N] \hat{\beta}} \hat{\gamma} \partial Z^{M} \bar{\partial} Z^{N}+\frac{1}{8} \alpha^{\prime} r \delta_{\hat{\gamma}}^{\hat{\beta}}
\end{gathered}
$$

imply from equation (65) that the Type II dilaton superfield $\Phi$ is related to the scale connections $\Omega_{P}^{(s)}$ and $\hat{\Omega}_{P}^{(s)}$ by

$$
D_{\alpha} \Phi=4 E_{\alpha}^{P} \Omega_{P}^{(s)}, \quad D_{\hat{\alpha}} \Phi=4 E_{\hat{\alpha}}^{P} \hat{\Omega}_{P}^{(s)} .
$$

One can check for the Type IIB superstring that (139) agrees with the relation found in equation (121), which confirms that the metric is in string gauge. Furthermore, the terms in (138) suggest that one should redefine the Type II three-form field strength as

$$
H_{P M N} \rightarrow H_{P M N}-\alpha^{\prime}\left(w_{P N M}^{(C S)}-\hat{w}_{P N M}^{(C S)}\right)
$$

\footnotetext{
${ }^{5}$ The triple pole of $\lambda^{\gamma} w_{\beta}$ with $T_{\lambda}$ can be computed using the formulas of [34] where $\lambda^{\alpha} w_{\alpha}=2 \alpha^{\prime} \partial h$ is the ghostnumber current, $T_{\lambda}=\frac{1}{10} N_{a b} N^{a b}-\frac{1}{2}(\partial h)^{2}-2 \partial^{2} h$ is the pure spinor stress tensor, and $h(y) h(z) \rightarrow-\log (y-z)$.
} 
where $w^{(C S)}$ is a Chern-Simons three-form constructed from the unhatted spin connections $\Omega_{P}^{(s)}$ and $\Omega_{P}^{a b}$, and $\hat{w}^{(C S)}$ is a Chern-Simons three-form constructed from the hatted spin connections $\hat{\Omega}_{P}^{(s)}$ and $\hat{\Omega}_{P}^{a b}$. However, since the differences of the vector components of the spin connections, $\Omega_{c}^{(s)}-\hat{\Omega}_{c}^{(s)}$ and $\Omega_{c}^{a b}-\hat{\Omega}_{c}^{a b}$, are expected to vanish on-shell, the vector components of the threeform, $H_{a b c}$, are not expected to be affected by (140).

It would be interesting to verify that these and other one-loop corrections to the BRST nilpotency and holomorphicity conditions are cancelled by the Fradkin-Tseytlin term and the Chern-Simons modifications to the three-form. It would also be interesting to verify that the sigma model actions of (24) and (56) are indeed conformally invariant at the quantum level when the background fields are on-shell.

\section{Acknowledgements:}

NB would like to thank CNPq grant 300256/94-9, Pronex grant 66.2002/1998-9, FAPESP grant 99/12763-0, and the Clay Mathematics Institute for partial financial support. The research of PSH was supported in part by PPARC SPG grant 613 . 


\section{References}

[1] N. Berkovits, S. Gukov and B.C. Vallilo, Superstrings in $2 D$ backgrounds with R-R flux and new extremal black holes, Nucl. Phys. B614 (2001) 195, hep-th/0107140.

[2] N. Berkovits, Covariant quantization of the Green-Schwarz superstring in a Calabi-Yau background, Nucl. Phys. B431 (1994) 258, hep-th/9404162; N. Berkovits and W. Siegel, Superspace effective actions for $4 D$ compactifications of heterotic and Type II superstrings, Nucl. Phys. B462 (1996) 213, hep-th/9510106; J. de Boer and K. Skenderis, Covariant computation of the low energy effective action of the heterotic superstring, Nucl. Phys. B481 (1996) 129, hep-th/9608078.

[3] N. Berkovits and C. Vafa, N=4 topological strings, Nucl. Phys. B433 (1995) 123, hepth/9407190; N. Berkovits, C. Vafa and E. Witten, Conformal Field Theory of AdS Background with Ramond-Ramond Flux, JHEP 03 (1999) 018, hep-th/9902098; N. Berkovits, Quantization of the Type II superstring in a curved six-dimensional background, Nucl. Phys. B565 (2000) 333, hep-th/9908041.

[4] P.S. Howe, H. Nicolai and A. Van Proeyen, Auxiliary fields and a superspace Lagrangian for linearised ten-dimensional supergravity, Phys. Lett. B112 (1982) 446.

[5] B.E.W. Nilsson and A. Tollsten, Supersymmetrisation of the $R^{4}$ term in superstring theories, Phys. Lett. B181 (1986) 63.

[6] C. Vafa and E. Witten, A one-loop test of string duality, Nucl. Phys. B447 (1995) 261, hep-th/9505053.

[7] A. Strominger, Loop corrections to the universal hypermultiplet, Phys. Lett. B421 (1998) 139, hep-th/9706195.

[8] M.B. Green and M. Gutperle, Effects of D-instantons, Nucl. Phys. B498 (1997) 195, hepth/9701093; M.B. Green, M. Gutperle and P. Vanhove, One loop in eleven dimensions, Phys. Lett. B409 (1997) 177, hep-th/9706175.

[9] A. Antoniadis, S. Ferrara, R. Minasian and K.S. Narain, $R^{4}$ couplings in $M$ - and type II theories on Calabi-Yau spaces, Nucl. Phys. B507 (1997) 571, hep-th/9707013.

[10] J.G. Russo and A.A. Tsetlin, One loop four graviton amplitude in eleven dimensional supergravity, Nucl. Phys. B508 (1997) 245, hep-th/9707134.

[11] N. Berkovits and C. Vafa, Type IIB $R^{4} H^{(4 g-4)}$ conjectures, Nucl. Phys. B533 (1998) 181, hep-th/9803145.

[12] M.B. Green and S. Sethi, Supersymmetry constraints on type IIB supergravity, Phys. Rev. D59 (1999) 046006, hep-th/9808061.

[13] M. Grisaru, A. van de Ven and D. Zanon, Four loop beta functions for the $N=1$ and $N=2$ supersymmetric non-linear sigma model in two dimensions, Phys. Lett. B173 (1986) 423. 
[14] E. Bergshoeff and M. de Roo, The quartic effective action of the heterotic string and supersymmtery, Nucl. Phys. B328 (1989) 439.

[15] M. de Roo, H. Suelmann and A. Wiedemann, The supersymmetric effective action of the heterotic string in ten dimensions, Nucl. Phys. B405 (1993) 326, hep-th/9210099.

[16] L. Bonora, M. Bregola, K. Lechner, P. Pasti and M. Tonin, Anomaly-free supergravity and super Yang-Mills theory in ten dimensions, Nucl.Phys. B296 (1988) 877; A discussion of the constraints in $N=1$ SUGRA-SYM in ten dimensions, Int. J. Mod. Phys. A5 (1990) 461.

[17] S. Bellucci and S.J. Gates, Jr., $D=10 N=1$ superspace supergravity and the Lorentz ChernSimons form, Phys. Lett. B208 (1988) 456.

[18] K. Peeters, P. Van Hove and A. Westerberg, Supersymmetric higher derivative actions in ten and eleven dimensions, the associated superalgebras and their formulation in superspace, Class. Quant. Grav. 18 (2001) 843, hep-th/0010167.

[19] M. Cederwall, U. Gran, M. Nielsen and B.E.W. Nilsson, Manifestly supersymmetric Mtheory, JHEP 10 (2000) 041, hep-th/0007035.

[20] S.J. Gates, Jr. and H. Nishino, Deliberations on 11D superspace for the M-theory effective action, Phys. Lett. B508 (2001) 155, hep-th/0101037.

[21] E. Witten, Twistor-like transform in ten dimensions, Nucl. Phys. B266 (1986) 245.

[22] J. J. Shapiro and C. Taylor, Supergravity torsion constraints from the 10D superparticle, Phys. Lett. B181 (1986) 67.

[23] I.L. Chau and B. Milewski, Light-like integrability and supergravity equations of motion in $D=10 N=1$ superspace, Phys. Lett. B216 (1989) 330.

[24] M. Grisaru, P.S. Howe, L. Mezincescu, B.E.W. Nilsson and P.K. Townsend, N=2 superstrings in a supergravity background, Phys. Lett. B162 (1985) 116.

[25] E. Bergshoeff, P.S. Howe, C.N. Pope, E. Sezgin and E. Sokatchev, Ten-dimensional supergravity from light-like integrability in loop superspace, Nucl. Phys. B354 (1991) 113.

[26] E. Bergshoeff, F. Delduc and E. Sokatchev, Lightlike integrability in loop superspace, KacMoody central charges and Chern-Simons terms, Phys. Lett. B262 (1991) 444.

[27] P.S. Howe, Pure spinor lines in superspace and ten-dimensional supersymmetric theories, Phys. Lett. B258 (1991) 141; P.S. Howe, Pure spinors, function superspaces and supergravity theories in ten and eleven dimensions, Phys. Lett. B273 (1991) 90.

[28] N. Berkovits, Covariant quantization of the superparticle using pure spinors, JHEP 09 (2001) 016, hep-th/0105050.

[29] N. Berkovits, Super-Poincaré covariant quantization of the superstring, JHEP 04 (2000) 018, hep-th/0001035; N. Berkovits, Covariant quantization of the superstring, Int. J. Mod. Phys. A16 (2001) 801, hep-th/0008145. 
[30] P.S. Howe and P.C. West, The complete $N=2$ D=10 supergravity, Nucl. Phys. B238 (1984) 181.

[31] P.S. Howe, Weyl superspace, Phys. Lett. B415 (1997) 149, hep-th/9707184.

[32] W. Siegel, Classical superstring mechanics, Nucl. Phys. B263 (1986) 93.

[33] J.L. Carr, S.J. Gates, Jr. and R.N. Oerter, $D=10 N=2 A$ supergravity in superspace, Phys. Lett. B189 (1987) 68.

[34] N. Berkovits, Relating the RNS and pure spinor formalisms for the superstring, JHEP 08 (2001) 026, hep-th/0104247.

[35] C.M. Hull and E. Witten, Supersymmetric sigma models and the heterotic string, Phys. Lett. B160 (1985) 398. 